\title{
MIMO-CDMA Systems: Signature and Beamformer Design With Various Levels of Feedback
}

\author{
Semih Serbetli, Member, IEEE, and Aylin Yener, Member, IEEE
}

\begin{abstract}
We investigate the signature and beamformer design problem for the uplink of multiple-input multiple-output code-division multiple-access (MIMO-CDMA) systems with the sum capacity and the systemwide mean-square error (MSE) as performance metrics. We first construct iterative algorithms to find the jointly optimum temporal signatures and transmit beamformers under the assumption of perfect temporal signature and transmit beamforming feedback. Next, motivated by the need to reduce the amount of feedback, we present a low-complexity sequential orthogonal temporal signature assignment algorithm for given transmit beamformers. Our approach is based on minimizing the difference between the performance of the MIMO-CDMA system and the described upper bounds at each signature assignment step. We next note that the transmit beamformers can be shaped depending on the channel state information (CSI) available at the transmitter. We investigate the cases of various levels of available feedback resulting in different beamformer structures and present a joint orthogonal temporal signature assignment and beamforming algorithm. We observe that as the available feedback level is increased, the performance of the joint signature and beamformer assignment algorithm approaches the performance upper bounds. In particular, we observe that a substantial performance gain is obtained when the individual channel state information is available at the transmitter side.
\end{abstract}

Index Terms-Mean-square error (MSE), multiple-input multiple-output code-division multiple-access (MIMO-CDMA) system, signature sequences, sum capacity, transmit beamforming.

\section{INTRODUCTION}

C ONSIDERING the rapidly increasing demand for high data rate and reliable wireless communications, high-capacity multiuser transmission schemes are of great importance for next generation wireless systems. Code-division multipleaccess (CDMA) systems emerge as promising candidates to meet these challenges. In addition, recent studies indicate that using multiple antennas at the transmitter and receiver can dramatically increase the performance of wireless communication systems [1]-[4]. It is well known that CDMA system performance is multiaccess interference limited and can be improved by efficient interference management techniques. To that end,

\footnotetext{
Manuscript received August 18, 2004; revised August 30, 2005. This work was supported in part by the National Science Foundation under CAREER Award 02-37727. This paper was presented in part at the Conference on Information Sciences and Systems (CISS'03), Baltimore, MD, March 2003, and CISS'04, Princeton, NJ, March 2004. The associate editor coordinating the review of this manuscript and approving it for publication was Prof. Gregori Vazquez.

S. Serbetli was with the Electrical Engineering Department, The Pennsylvania State University, University Park, PA 16802 USA. He is now with Philips Research, Eindhoven, The Netherlands (e-mail: semih.serbetli@philips.com).

A. Yener is with the Electrical Engineering Department, The Pennsylvania State University, University Park, PA 16802 USA (e-mail: yener@ee.psu.edu). Digital Object Identifier 10.1109/TSP.2006.874787
}

multiuser detection and interference avoidance exploit the temporal structure of the received signal [5]-[7]. Recent literature has suggested that, the use of multiple antennas for CDMA systems provides additional interference suppression by exploiting the spatial structure of the system [8]-[10].

The uplink performance of a multiuser system can be improved significantly by carefully adopting the transmitters of each user. Optimum or near-optimum transmit strategies have been investigated for multiuser systems with various performance metrics up to date. For single-antenna systems, information theoretic sum capacity and user capacity of a CDMA cell, and iterative algorithms that converge to the optimum transmitters, i.e., signature sequence sets, are given in [6], [11]-[14]. Similarly, for narrowband multiuser multiple-input multiple-output (MIMO) systems, transmitters have been optimized with respect to sum capacity and the mean-square error (MSE) [15], [16].

In the case of a CDMA system where each user and the base station are equipped with multiple antennas, termed hereafter a MIMO-CDMA system, users' transmissions can be coordinated both temporally and spatially. Transmitter design, in this case, entails judicious choice of temporal signatures and transmit beamformers. Most of the transmission schemes previously proposed for MIMO-CDMA systems rely on assigning orthogonal signature sequences to the antennas/symbols without reusing the signatures for different users, or integrating space-time codes to the system [17], [18].

The premise of this paper is that, as in the case of single-antenna CDMA systems, and narrowband multiuser MIMO systems, we can utilize the channel state related feedback at the transmitter side to substantially improve the uplink performance of a MIMO-CDMA system, where each user is eventually assigned a unique signature sequence, and a transmit beamformer. Our objective in this paper is to design the appropriate transmit beamformers and signatures considering the sum capacity, and the systemwide mean squared error (MSE) as the performance metrics. We first investigate the unlimited reliable feedback case and construct iterative algorithms to find the optimum transmit beamformer and signature set. Motivated by milder feedback requirements, we constrain the signature set to an orthogonal set and investigate the joint orthogonal signature assignment and transmit beamformer design. In contrast to references [17], [18], in this paper, we consider an overloaded system where the orthogonal signatures are reused by several users and such users interfere with each other. In such a scenario, efficient grouping of the users that will share the same orthogonal signatures, and designing the beamformer with available feedback for each user, are new design problems that we encounter. We present a low-complexity orthogonal temporal signature assignment algorithm that aims to approach 


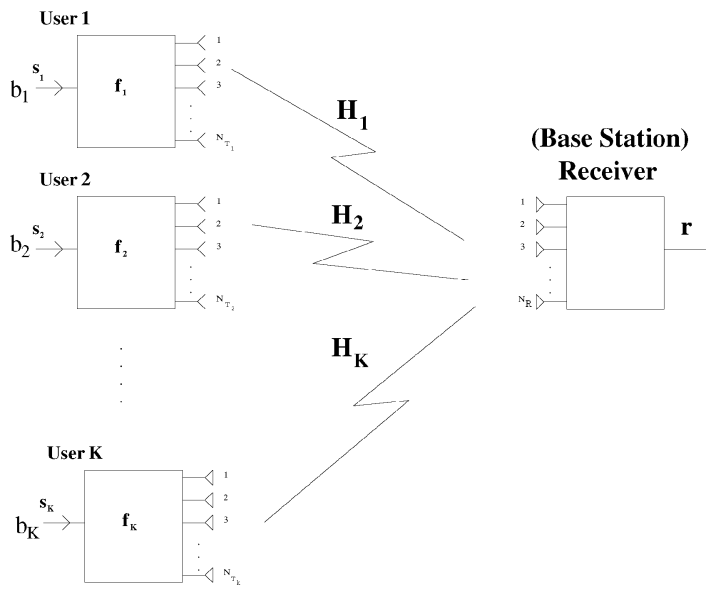

Fig. 1. MIMO-CDMA system model.

the performance bounds for given transmit beamformers. Since the performance of the orthogonal temporal signature assignment algorithm is a function of the transmit beamformers of the users, we next tackle the problem of designing the transmit beamformers for different levels of feedback available at the transmit side of each user, and combine the proposed orthogonal temporal signature assignment algorithm with perfectly controlled transmit beamforming, eigentransmit beamforming and antenna selection. Throughout the paper, we first develop signature and beamformer design algorithms for single-path synchronous channels, and then extend our results for multipath and asynchronous channels. Additionally, to provide a performance benchmark for the proposed algorithms, we develop an upper bound for the sum capacity and a lower bound for the MSE. We observe that, for both metrics, joint design of transmit beamformers and signatures results in performance that approaches to the described performance bounds with increased level of available feedback at the transmit side.

The organization of the paper is as follows. The system model is given in Section II. The performance metrics and the corresponding performance bounds are formulated in Section III. The signature and beamformer design algorithm with perfect feedback is proposed in Section IV. The orthogonal temporal signature assignment algorithm for given transmit beamformers is presented in Section V. In Section VI, the impact of transmit beamformers is investigated, and the joint transmit beamformer and temporal signature selection methods are proposed for different feedback levels. Numerical results demonstrating performance of the algorithms are presented in Section VII. Section VIII concludes the paper.

\section{SySTEM MODEL AND ASSUMPTIONS}

We consider the uplink of a single-cell MIMO-CDMA system with $K$ users and processing gain $N$. The common receiver is equipped with $N_{R}$ receive antennas. User $k$ has $N_{T_{k}}$ transmit antennas, and unit energy signature waveform $s_{k}(t)$. User $k$ transmits its symbol, $b_{k}$, by precoding it with an $N_{T_{k}} \times 1$ unitnorm transmit beamforming vector, $\mathbf{f}_{k}$ (Fig. 1), and it passes through a multipath channel with an impulse response

$$
\mathbf{H}_{k}(t)=\sum_{l=1}^{L} \mathbf{H}_{k, l} \delta\left(t-\tau_{k, l}-v_{k}\right)
$$

where $\mathbf{H}_{k, l}, \tau_{k, l}$ and $v_{k}$ are the complex MIMO channel and the delay associated with the $l$ th path of the $k$ th user and the delay of user $k$, respectively. For simplicity, each user's channel is assumed to be composed of exactly $L$ paths and the delays are assumed to be chip synchronous i.e., $\tau_{k, l}=(l-1) T_{c}$ and $v_{k}=n T_{c}$. Without loss of generality, the asynchronous multipath channel can be modeled with $\widetilde{L}=L+\max _{k}\left(\left\{v_{k}\right\}\right)$ paths for each user. The received signal is chip matched filtered and sampled at the chip rate over the entire observation interval of $(N+\widetilde{L}-1)$ chips. MIMO channels and the temporal signatures of the users provide effective spatial temporal signatures, $\left\{\mathbf{t}_{k}\right\}_{k=1}^{K}$, for the users. Assuming $\widetilde{L} \ll N$ and using the multipath model in [7], the effective spatial temporal signature of user $k, \mathbf{t}_{k}$, can be expressed

$$
\begin{aligned}
\mathbf{t}_{k} & =\mathbf{A}_{k} \mathbf{s}_{k}=\mathbf{Q}_{k} \mathbf{f}_{k} \\
\text { with } \mathbf{A}_{k} & =\left[\begin{array}{ccccc}
\sqrt{P_{k}} \mathbf{H}_{k, 1} \mathbf{f}_{k} & 0 & \cdots & 0 & 0 \\
\sqrt{P_{k}} \mathbf{H}_{k, 2} \mathbf{f}_{k} & \sqrt{P_{k}} \mathbf{H}_{k, 1} \mathbf{f}_{k} & 0 & \cdots & 0 \\
\vdots & \vdots & \ddots & \ddots & \vdots \\
0 & 0 & \cdots & 0 & \sqrt{P_{k}} \mathbf{H}_{k, \widetilde{L}} \mathbf{f}_{k}
\end{array}\right] \\
\mathbf{Q}_{k} & {\left[\begin{array}{c}
\sqrt{P_{k}} \mathbf{H}_{k, 1}\left(\mathbf{s}_{k}\right)_{1} \\
\sqrt{P_{k}} \mathbf{H}_{k, 3}\left(\mathbf{s}_{k}\right)_{1}+\sqrt{P_{k}} \mathbf{H}_{k, 2}\left(\mathbf{s}_{k}\right)_{2}+\sqrt{P_{k}} \mathbf{H}_{k, 1}\left(\mathbf{s}_{k}\right)_{3} \\
\vdots \\
\sqrt{P_{k}} \mathbf{H}_{k, 2}\left(\mathbf{s}_{k}\right)_{1}+\sqrt{P_{k}} \mathbf{H}_{k, 1}\left(\mathbf{s}_{k}\right)_{2} \\
\sqrt{P_{k}} \mathbf{H}_{k, \widetilde{L}}\left(\mathbf{s}_{k}\right)_{N}
\end{array}\right.}
\end{aligned}
$$

where $\mathbf{s}_{k}$ is the chip matched filtered and sampled form of $s_{k}(t)$, and $P_{k}$ is the transmit power of user $k$.

Throughout the paper, we assume that the transmit beamforming vector for each user is given and fixed first. We relax this assumption later and investigate the effect of transmit beamforming on the system. The received vector at the common destination is given by

$$
\mathbf{r}=\sum_{j=1}^{K} \mathbf{t}_{j} b_{j}+\mathbf{n}
$$

where $\mathbf{n}$ is the zero-mean complex Gaussian noise vector with $E\left[\mathbf{n n}^{H}\right]=\sigma^{2} \mathbf{I}$, and $(\cdot)^{H}$ denotes the Hermitian of a vector or matrix. We assume that the channel realizations of each path and delays of the users are constant and perfectly known at the receiver side. Despite the fact that (4) is a general model for asynchronous multipath channels, it adds little insight in terms of the performance and complicates the derivations. Thus, in the sequel, we will first assume synchronous users with single-path channels, i.e.,

$$
\mathbf{A}_{j}=\left[\begin{array}{cccc}
\mathbf{a}_{j} & \mathbf{0} & \cdots & \mathbf{0} \\
\mathbf{0} & \mathbf{a}_{j} & \vdots & \vdots \\
\vdots & \vdots & \ddots & \mathbf{0} \\
\mathbf{0} & \mathbf{0} & \cdots & \mathbf{a}_{j}
\end{array}\right] ; \quad \mathbf{Q}_{j}=\left[\begin{array}{c}
\sqrt{P_{j}} \mathbf{H}_{j}\left(\mathbf{s}_{j}\right)_{1} \\
\sqrt{P_{j}} \mathbf{H}_{j}\left(\mathbf{s}_{j}\right)_{2} \\
\vdots \\
\sqrt{P_{j}} \mathbf{H}_{j}\left(\mathbf{s}_{j}\right)_{N}
\end{array}\right] ;
$$


with $\mathbf{a}_{j}=\sqrt{P_{j}} \mathbf{H}_{j} \mathbf{f}_{j}$, where $\mathbf{H}_{j}$ is the single-path MIMO channel of user $j$. We will extend the results to multipath and asynchronous channels, at the conclusion of each section.

Our aim in this work is to design transmit beamformer and signature sets that will optimize the performance metric chosen for MIMO-CDMA systems with the number of users exceeding the dimensionality of the temporal signal space, i.e., systems with $N<K \leq N N_{R}$ users.

\section{Performance Metrics}

We consider two systemwide performance metrics: the sum capacity of and the total incurred MSE in a MIMO-CDMA cell. Both metrics have been considered for single-antenna CDMA and multiuser MIMO systems [12], [15], [16], [19], and are equally meaningful metrics representing the system performance: the former representing the information theoretic achievable sum rate of all users, and the latter involving the employment of linear receivers and is the sum of the MSE incurred by each user in the system. Therefore, we will consider the joint design of temporal signatures and transmit beamformers of all users in the cell with aim of i) maximizing the information theoretic sum capacity, or ii) minimizing the total MSE with each user employing a linear receiver.

\section{A. Sum Capacity}

The sum capacity of a multiuser system with effective signatures $\left\{\mathbf{t}_{j}=\mathbf{A}_{j} \mathbf{s}_{j}\right\}$ is given by [11]:

$$
C_{\text {sum }}=\frac{1}{2} \log \left[\operatorname{det}\left(\mathbf{I}_{N N_{R}}+\sigma^{-2} \sum_{j=1}^{K} \mathbf{t}_{j} \mathbf{t}_{j}^{H}\right)\right] .
$$

The sum capacity optimization problem (OP1) becomes

$$
\begin{aligned}
& \max _{\left\{\left\{\mathbf{s}_{j}\right\},\left\{\mathbf{f}_{j}\right\}\right\}} C_{\text {sum }}=\frac{1}{2} \log \left[\operatorname{det}\left(\mathbf{I}_{N N_{R}}+\sigma^{-2} \sum_{j=1}^{K} \mathbf{t}_{j} \mathbf{t}_{j}^{H}\right)\right] \\
& \text { s.t. }\left\|\mathbf{s}_{j}\right\|=1, \quad\left\|\mathbf{f}_{j}\right\|=1, \quad j=1, \ldots, K
\end{aligned}
$$

where $\mathbf{t}_{j}$ is a function of $\mathbf{s}_{j}$ and $\mathbf{f}_{j}$.

\section{B. Systemwide MSE}

The systemwide MSE with effective signatures $\left\{\mathbf{t}_{j}=\right.$ $\left.\mathbf{A}_{j} \mathbf{s}_{j}\right\}_{j=1}^{K}$ and linear receivers $\left\{\mathbf{g}_{j}\right\}_{j=1}^{K}$ is

$$
\begin{aligned}
\mathrm{MSE} & =\sum_{j=1}^{K} E\left[\left\|\mathbf{g}_{j}^{H} \mathbf{r}-b_{j}\right\|^{2}\right] \\
& =\sum_{j=1}^{K}\left(\sum_{i=1}^{K} \mathbf{t}_{i}^{H} \mathbf{g}_{j} \mathbf{g}_{j}{ }^{H} \mathbf{t}_{i}-\mathbf{t}_{j}^{H} \mathbf{g}_{j}-\mathbf{g}_{j}{ }^{H} \mathbf{t}_{j}+1+\sigma^{2} \mathbf{g}_{j}{ }^{H} \mathbf{g}_{j}\right)
\end{aligned}
$$

For given effective signatures $\left\{\mathbf{t}_{j}\right\}_{j=1}^{K}$, the receivers minimizing the MSE results in the well-known receivers':

\footnotetext{
${ }^{1}$ Throughout the paper, ()$^{\dagger}$ should be substituted for ()$^{-1}$ if an inversion problem arises for an interference limited system, i.e., $\sigma^{2} \rightarrow 0$.
}

$\left\{\mathbf{g}_{j}=\left(\sum_{i=1}^{K} \mathbf{t}_{i} \mathbf{t}_{i}^{H}+\sigma^{2} \mathbf{I}\right)^{-1} \mathbf{t}_{j}\right\}_{j=1}^{K} \quad$ [20]. When these receivers are employed, the MSE is [16]

MSE $=K-N N_{R}+\sigma^{2} \operatorname{tr}\left\{\left(\sigma^{2} \mathbf{I}_{N N_{R}}+\sum_{j=1}^{K} \mathbf{t}_{j} \mathbf{t}_{j}{ }^{H}\right)^{-1}\right\}_{(9)}$

The MSE optimization problem (OP2) becomes

$$
\begin{aligned}
& \min _{\left\{\left\{\mathbf{s}_{j}\right\},\left\{\mathbf{f}_{j}\right\}\right\}} \operatorname{MSE}= K-N N_{R} \\
&+\sigma^{2} \operatorname{tr}\left\{\left(\sigma^{2} \mathbf{I}_{N N_{R}}+\sum_{j=1}^{K} \mathbf{t}_{j} \mathbf{t}_{j}{ }^{H}\right)^{-1}\right\} \\
& \text { s.t. } \quad\left\|\mathbf{s}_{j}\right\|=1, \quad\left\|\mathbf{f}_{j}\right\|=1, \quad j=1, \ldots, K .
\end{aligned}
$$

It is easily seen that, for both $\mathrm{OP} 1$ and $\mathrm{OP} 2$, the performance is a function of the effective signatures $\left\{\mathbf{t}_{j}=\mathbf{A}_{j} \mathbf{s}_{j}\right\}_{j=1}^{K}$ and the subspace of the effective signatures is defined by the span of matrices $\left\{\mathbf{A}_{j}\right\}_{j=1}^{K}$. The resulting dimension of the users' signature space is on the order of the number of receivers multiplied by $N$. However, each user's signature space is constrained by its channel realization. Transmitter optimization in this case entails optimization of temporal signatures and transmit beamformers given the channels of the users. The resulting optimum transmitters are a function of the particular channel realizations of the users, and do not have a closed form. However, as is explained below, we can easily construct performance upper bounds. Given the hardship of the optimization problem at hand, it is meaningful to try to construct algorithms such that the performance of the resulting temporal signatures and the transmit beamformers approach the performance upper bounds, and hence the actual optimum performance.

We now explain how we obtain the performance bounds. We first note that, from a system point of view, the MIMO-CDMA system can be viewed as a CDMA system with a processing gain $N N_{R}$ with structural constraints on the effective signatures. The received power of user $j$ is

$$
P_{\text {rec }, j}=\mathbf{t}_{j}^{H} \mathbf{t}_{j}=\mathbf{s}_{j}^{H} \mathbf{A}_{j}^{H} \mathbf{A}_{j} \mathbf{s}_{j} .
$$

For a single-path synchronous MIMO-CDMA system with unit-norm temporal signatures (11) simplifies to $P_{\text {rec }, j}=\left\|\mathbf{a}_{j}\right\|^{2}=P_{j}\left\|\mathbf{H}_{j} \mathbf{f}_{j}\right\|^{2}$. Thus, the performance of a single-antenna CDMA system with processing gain $N N_{R}$, and received powers $\left\{\left\|\mathbf{a}_{j}\right\|^{2}\right\}$, forms an upper bound for the performance of the MIMO-CDMA system. For single-antenna, single-cell CDMA systems, the optimum signatures for maximizing sum capacity and minimizing the MSE coincide [14]. For $N<K \leq N N_{R}$, the performance upper bound is simply the performance of an underloaded CDMA system with processing gain $N N_{R}$, and orthogonal effective signature sequences are optimum [12]. The resulting sum capacity and the MSE values yield our performance upper bounds. In particular, the sum capacity upper bound, $C_{\text {upper }}$ is [12]

$$
C_{\text {sum }} \leq C_{\text {upper }}=\frac{1}{2} \sum_{j=1}^{K} \log \left[1+\sigma^{-2}\left\|\mathbf{a}_{j}\right\|^{2}\right]
$$


and the systemwide MSE lower bound, $\mathrm{MSE}_{\text {lower }}$ is [19]

$$
\mathrm{MSE} \geq \mathrm{MSE}_{\text {lower }}=\sum_{j=1}^{K}\left(\frac{\sigma^{2}}{\sigma^{2}+\left\|\mathbf{a}_{j}\right\|^{2}}\right)
$$

Note that the performance metrics in (6) and (9) and performance bounds in (12) and (13) are for single-path synchronous channels. Using the model in (2) and (3), the sum capacity and the MSE expressions of asynchronous multipath channels can be found by replacing the term $N$ in (6), (9) with $\widetilde{N}=N+\widetilde{L}-1$, and the bounds are

$$
\begin{gathered}
C_{\text {sum }} \leq C_{\text {upper }}=\frac{1}{2} \sum_{j=1}^{K} \log \left[1+\frac{P_{\text {rec }, j}}{\sigma^{2}}\right] \\
\mathrm{MSE} \geq \mathrm{MSE}_{\text {lower }}=\sum_{j=1}^{K}\left(\frac{\sigma^{2}}{\sigma^{2}+P_{\text {rec }, j}}\right) .
\end{gathered}
$$

\section{Effect of Imperfect Channel State Information}

Throughout our formulation of the performance metrics, we have assumed that the receiver has perfect channel state information (CSI) of all users. In practical scenarios, CSI errors will be present and should be taken into account in system design. The effect of imperfect CSI on the sum capacity and the MSE of a MIMO link is studied in references [21]-[23]. [21] shows that, when estimation errors exist, the capacity has a lower bound, and the channel estimation error acts like additional Gaussian noise. [23] shows that the effect of CSI errors can be reflected in transceiver design via additional Gaussian noise with total MSE as the performance metric. Thus, the formulation of the performance metrics and following development for both sum capacity and the systemwide MSE performance metrics can be adapted to the case of imperfect CSI by simply considering the channel estimation errors as a source of additive Gaussian noise, reducing the effective signal-to-noise ratio (SNR) of each user. In the sequel, for clarity of exposition, we will assume that the errors in CSI is already reflected in our designs as additional Gaussian noise.

In the following sections, we pose the problem of optimizing the performance in terms of sum capacity and MSE in the presence of various levels of feedback and devise iterative algorithms. The performance bounds, $C_{\text {upper }}$ and $\mathrm{MSE}_{\text {lower }}$ will be used as benchmarks to evaluate the performance of the algorithms.

We reiterate that throughout the rest of the paper, the performance metrics as well as the algorithms are formulated first for the synchronous single-path uplink of a MIMO-CDMA cell. This assumption is valid, for instance, when the channel gain of one path is comparatively large than the others and the delays of users are small with respect to the processing gain. The development is then extended to address the multipath and asynchronous channel models using the general channel model in (2)-(3) at the conclusion of each subsection.

\section{SigNATURE AND BEAMFORMER DESIGN WITH PERFECT FEEDBACK}

Our aim in this section is to design temporal signature and transmit beamformer sets that achieve performances near the performance bounds developed in the previous section. We assume that we have a feedback channel to the transmitter side where the signatures and beamformers obtained for each user can be communicated exactly. Here after, we will term this scenario the perfect feedback case.

\section{A. Sum Capacity}

Recall that the sum capacity of the MIMO-CDMA system is

$$
C_{\text {sum }}=\frac{1}{2} \log \left[\operatorname{det}\left(\mathbf{I}_{N N_{R}}+\sigma^{-2} \sum_{j=1}^{K} \mathbf{t}_{j} \mathbf{t}_{j}^{H}\right)\right] .
$$

Using the properties $\operatorname{det}(\mathbf{X Y})=\operatorname{det}(\mathbf{X}) \operatorname{det}(\mathbf{Y})$ for square matrices and $\operatorname{det}\left(\mathbf{I}_{d \times d}+\Phi_{d \times j} \Xi_{j \times d}\right)=\operatorname{det}\left(\mathbf{I}_{j \times j}+\Xi_{j \times d} \Phi_{d \times j}\right)$ for rectangular matrices $\Xi j \times d$ and $\Phi_{d \times j}$, the sum capacity can be expressed in terms of user $k$ 's parameters as

$$
\begin{aligned}
C_{\text {sum }} & =\frac{1}{2} \log \left[\operatorname{det}\left(\sigma^{-2}\left(\mathbf{t}_{k} \mathbf{t}_{k}^{H}+\mathbf{E}_{k}\right)\right)\right] \\
& =\frac{1}{2} \log \left[\operatorname{det}\left(\sigma^{-2} \mathbf{E}_{k}\right)\right]+\frac{1}{2} \log \left[1+\left(\mathbf{t}_{k}^{H} \mathbf{E}_{k}^{-1} \mathbf{t}_{k}\right)\right] \\
& =\gamma_{k}+\frac{1}{2} \log \left[1+Z_{k}^{\text {sum }}\left(\mathbf{f}_{k}, \mathbf{s}_{k}\right)\right]
\end{aligned}
$$

where $\mathbf{E}_{k}=\sum_{i=1, i \neq k}^{K} \mathbf{t}_{i} \mathbf{t}_{i}^{H}+\sigma^{2} \mathbf{I}_{N N_{R}}$ is the interference covariance matrix of user $k, \gamma_{k}$ represents the terms independent of user $k$, and $Z_{k}^{\text {sum }}\left(\mathbf{f}_{k}, \mathbf{s}_{k}\right)=\mathbf{t}_{k}^{H} \mathbf{E}_{k}^{-1} \mathbf{t}_{k}$ is the term denoting the effect of user $k$ on the sum capacity. Recalling that $\mathbf{t}_{k}=\mathbf{A}_{k} \mathbf{s}_{k}=\mathbf{Q}_{k} \mathbf{f}_{k}$, we can express $Z_{k}^{\text {sum }}\left(\mathbf{f}_{k}, \mathbf{s}_{k}\right)$ as

$$
Z_{k}^{\text {sum }}\left(\mathbf{f}_{k}, \mathbf{s}_{k}\right)=\mathbf{s}_{k}^{H} \mathbf{A}_{k}^{H} \mathbf{E}_{k}^{-1} \mathbf{A}_{k} \mathbf{s}_{k}=\mathbf{f}_{k}^{H} \mathbf{Q}_{k}^{H} \mathbf{E}_{k}^{-1} \mathbf{Q}_{k} \mathbf{f}_{k}
$$

Thus, the sum capacity can be expressed in terms of the signature of user $k, \mathbf{s}_{k}$, as

$$
C_{\text {sum }}=\gamma_{k}+\frac{1}{2} \log \left[1+\mathbf{s}_{k}^{H} \mathbf{A}_{k}^{H} \mathbf{E}_{k}^{-1} \mathbf{A}_{k} \mathbf{s}_{k}\right]
$$

and in terms of the transmit beamformer of user $k, \mathbf{f}_{k}$, as

$$
C_{\text {sum }}=\gamma_{k}+\frac{1}{2} \log \left[1+\mathbf{f}_{k}^{H} \mathbf{Q}_{k}^{H} \mathbf{E}_{k}^{-1} \mathbf{Q}_{k} \mathbf{f}_{k}\right]
$$

We will use (19) and (20) in the construction of the iterative algorithm to find the capacity maximizing signatures and beamformers.

\section{B. Systemwide MSE}

Let us now follow the same approach for the systemwide MSE. Recall that the MSE of the MIMO-CDMA system with effective signatures $\left\{\mathbf{t}_{k}\right\}$ and the corresponding optimum linear MMSE receivers is

$$
\mathrm{MSE}=K-N N_{R}+\sigma^{2} \operatorname{tr}\left\{\mathrm{T}^{-1}\right\}
$$


where $\mathbf{T}=\sigma^{2} \mathbf{I}+\sum_{j=1}^{K} \mathbf{t}_{j} \mathbf{t}_{j}{ }^{H}$. Using matrix inversion lemma, $\mathbf{T}^{-1}$ can be expressed as

$$
\mathbf{T}^{-1}=\mathbf{E}_{k}{ }^{-1}-\frac{\mathbf{E}_{k}{ }^{-1} \mathbf{t}_{k} \mathbf{t}_{k}{ }^{H} \mathbf{E}_{k}{ }^{-1}}{1+\mathbf{t}_{k}{ }^{H} \mathbf{E}_{k}{ }^{-1} \mathbf{t}_{k}} .
$$

In this form, the MSE can be expressed as a function of parameters of user $k$, plus the contributions of the remaining users as follows:

$$
\begin{aligned}
\mathrm{MSE} & =K-N N_{R}+\sigma^{2} \operatorname{tr}\left\{\mathbf{E}_{k}{ }^{-1}-\frac{\mathbf{E}_{k}{ }^{-1} \mathbf{t}_{k} \mathbf{t}_{k}{ }^{H} \mathbf{E}_{k}{ }^{-1}}{1+\mathbf{t}_{k}{ }^{H} \mathbf{E}_{k}{ }^{-1} \mathbf{t}_{k}}\right\} \\
& =\beta_{k}-\sigma^{2} \frac{\mathbf{t}_{k}{ }^{H} \mathbf{E}_{k}{ }^{-2} \mathbf{t}_{k}}{1+\mathbf{t}_{k}{ }^{H} \mathbf{E}_{k}{ }^{-1} \mathbf{t}_{k}}=\beta_{k}-\sigma^{2} Z_{k}^{\mathrm{MSE}}\left(\mathbf{f}_{k}, \mathbf{s}_{k}\right)
\end{aligned}
$$

where $\beta_{k}$ represents terms independent of user $k$, and $Z_{k}^{\mathrm{MSE}}\left(\mathbf{f}_{k}, \mathbf{s}_{k}\right)$ is the term denoting the effect of user $k$ on the MSE. Once again, using $\mathbf{t}_{k}=\mathbf{A}_{k} \mathbf{s}_{k}=\mathbf{Q}_{k} \mathbf{f}_{k}$, we can easily express $Z_{k}^{\mathrm{MSE}}\left(\mathbf{f}_{k}, \mathbf{s}_{k}\right)$ as

$$
\begin{aligned}
Z_{k}^{\mathrm{MSE}}\left(\mathbf{f}_{k}, \mathbf{s}_{k}\right) & =\frac{\mathbf{s}_{k}{ }^{H} \mathbf{A}_{k}{ }^{H} \mathbf{E}_{k}^{-2} \mathbf{A}_{k} \mathbf{s}_{k}}{\mathbf{s}_{k}{ }^{H}\left(\mathbf{I}+\mathbf{A}_{k}{ }^{H} \mathbf{E}_{k}^{-1} \mathbf{A}_{k}\right) \mathbf{s}_{k}} \\
& =\frac{\mathbf{f}_{k}{ }^{H} \mathbf{Q}_{k}{ }^{H} \mathbf{E}_{k}^{-2} \mathbf{Q}_{k} \mathbf{f}_{k}}{\mathbf{f}_{k}{ }^{H}\left(\mathbf{I}+\mathbf{Q}_{k}{ }^{H} \mathbf{E}_{k}^{-1} \mathbf{Q}_{k}\right) \mathbf{f}_{k}} .
\end{aligned}
$$

Consequently, the total MSE, in terms of the signature of user $k, \mathbf{s}_{k}$, can be expressed as

$$
\text { MSE }=\beta_{k}-\sigma^{2}\left(\frac{\mathbf{s}_{k}{ }^{H} \mathbf{A}_{k}{ }^{H} \mathbf{E}_{k}^{-2} \mathbf{A}_{k} \mathbf{s}_{k}}{\mathbf{s}_{k}{ }^{H}\left(\mathbf{I}+\mathbf{A}_{k}{ }^{H} \mathbf{E}_{k}^{-1} \mathbf{A}_{k}\right) \mathbf{s}_{k}}\right)
$$

and, in terms of the transmit beamformer of user $k, \mathbf{f}_{k}$, as

$$
\text { MSE }=\beta_{k}-\sigma^{2}\left(\frac{\mathbf{f}_{k}{ }^{H} \mathbf{Q}_{k}{ }^{H} \mathbf{E}_{k}^{-2} \mathbf{Q}_{k} \mathbf{f}_{k}}{\mathbf{f}_{k}{ }^{H}\left(\mathbf{I}+\mathbf{Q}_{k}{ }^{H} \mathbf{E}_{k}^{-1} \mathbf{Q}_{k}\right) \mathbf{f}_{k}}\right) .
$$

\section{Iterative Algorithms}

Equations (25) and (26), as well as (19) and (20), suggest an iterative way of improving the performance. Specifically, iterative algorithms that maximize the performance at each step can be devised by optimizing the temporal signature and transmit beamformer of one user, say user $k, \mathbf{s}_{k}$ and $\mathbf{f}_{k}$, at each step. From the perspective of user $k$, the performance can be improved by choosing $\mathbf{s}_{k}$ and $\mathbf{f}_{k}$ to maximize the second terms in (19) and (20), in terms of the sum capacity, and the second terms in (25) and (26), in terms of the MSE. Sum capacity improvement is accomplished by choosing $\mathbf{s}_{k}$ to be the eigenvector which corresponds to the maximum eigenvalue of $\mathbf{A}_{k}^{H} \mathbf{E}_{k}^{-1} \mathbf{A}_{k}$ in (19), and $\mathbf{f}_{k}$ to be the eigenvector which corresponds to the maximum eigenvalue of $\mathbf{Q}_{k}^{H} \mathbf{E}_{k}^{-1} \mathbf{Q}_{k}$ in (20), i.e., maximizing $Z_{k}^{\text {sum }}\left(\mathbf{f}_{k}, \mathbf{s}_{k}\right)$ in (18). Similarly, $Z_{k}^{\mathrm{MSE}}\left(\mathbf{f}_{k}, \mathbf{s}_{k}\right)$ in (24) can be

\begin{tabular}{|c|c|}
\hline End & $\begin{array}{l}\text { Updating signatures and beamformers in step }(n+1) \\
k=1: K \\
\mathbf{E}_{k}(n+1)=\sum_{i<k} \mathbf{t}_{i}(n+1) \mathbf{t}_{i}(n+1)^{H}+\sum_{i>k} \mathbf{t}_{i}(n) \mathbf{t}_{i}(n)^{H}+\sigma^{2} \mathbf{I} \\
\mathbf{s}_{k}(n+1)=\underset{\mathbf{s}_{k} \in C^{N} ; \mathbf{s}_{k}^{H} \mathbf{s}_{k}=1}{\arg \max Z_{k}^{\star}(n+1)} \\
\text { Update } \mathbf{Q}_{k}(n+1) \\
\mathbf{f}_{k}(n+1)=\underset{\mathbf{f}_{k} \in C^{N} ; \mathbf{f}_{k}^{H} \mathbf{f}_{k}=1}{\arg \max } Z_{k}^{\star}(n+1) \\
\text { Update } \mathbf{A}_{k}(n+1) \\
\mathbf{t}_{k}(n+1)=\mathbf{A}_{k}(n+1) \mathbf{s}_{k}(n+1) \\
\star \text { : sum or MSE }\end{array}$ \\
\hline
\end{tabular}
maximized by choosing $\mathbf{s}_{k}$ to be the generalized eigenvector which corresponds to the maximum generalized eigenvalue of $\left(\mathbf{A}_{k}{ }^{H} \mathbf{E}_{k}^{-2} \mathbf{A}_{k}\right)$ and $\left(\mathbf{I}+\mathbf{A}_{k}{ }^{H} \mathbf{E}_{k}^{-1} \mathbf{A}_{k}\right)$ in (25), and choosing $\mathbf{f}_{k}$ to be the generalized eigenvector which corresponds to the
TABLE I

SignATURE AND BEAMFORMER DESIGN With PERFECT FEEDBACK

maximum generalized eigenvalue of $\left(\mathbf{Q}_{k}{ }^{H} \mathbf{E}_{k}^{-2} \mathbf{Q}_{k}\right)$ and $(\mathbf{I}+$ $\left.\mathbf{Q}_{k}{ }^{H} \mathbf{E}_{k}^{-1} \mathbf{Q}_{k}\right)$ in (26). First, each user is assigned a random signature sequence and a beamformer. Next, the two iterative algorithms constructed to improve the sum capacity and the MSE, iterate over the users, optimizing the signature and then the beamformer (or vice versa) of each user while all other parameters are fixed, improving the performance at each step and yielding a monotonic sequence of performance values. This implies that the algorithms, which produce monotonic sequences that are bounded by the corresponding performance bound, i.e., (12) or (13), are convergent [24]. A formal proof that the algorithms converge to the global optimum point eludes us due to the nonconvexity of the problem, however, our numerical result will show that the performance of the algorithms consistently comes very close to the performance bounds given in Section III.

We term this class of algorithms, whose outline we present in Table I, signature and beamformer design with perfect feedback (PF). Note that, in Table I, we can obtain the particular algorithm according to $C_{\text {sum }}$ or MSE, by replacing $Z_{k}^{\star}$ with $Z_{k}^{\text {sum }}$ or $Z_{k}^{\mathrm{MSE}}$ respectively. Note also that the update mechanisms do not depend which particular definitions of $\mathbf{A}_{k}$ and $\mathbf{Q}_{k}$, i.e., (3) or (5), are used. Thus, the algorithm can be implemented for both single-path synchronous channels as well as multipath and asynchronous channels. The algorithms proposed in Table I can be run online or offline. Online implementation of the algorithms requires the feedback of the temporal signature and beamformer information to the users at each step whereas the offline implementation requires the feedback of the resulting optimum temporal signature and beamformer of each user to the user.

\section{Orthogonal Temporal Signature Assignment}

In the previous section, we proposed iterative algorithms to design temporal signatures and transmit beamformers that improve the performance of the MIMO-CDMA system at each step. It is important to note that this approach eventually requires perfect feedback of the resulting signatures and transmit beamformers to the transmitter side. The resulting signatures and transmit beamformers are a function of the channel parameters. This implies that the algorithm would have to be run each time the channel changes. In practice, such amount of feedback may be overwhelming, rendering the implementation impractical. Hence, it may be necessary to look for ways to reduce the 
feedback requirement in exchange for a modest sacrifice in performance.

An effective way to significantly reduce the amount of feedback to the transmitter side, which we will follow for the remainder of this paper, is to constrain the temporal signature set to be a set of orthogonal signatures. Because we have $K>N$ users in the system, we will assume the largest such signature set, i.e., $N$ orthogonal signatures. In contrast to [17] and [18], the temporal signatures will be reused between the users up to $N_{R}$ times since $N<K \leq N N_{R}$. Users transmitting with the same signature will be distinguished via the spatial signatures. In this case, since each orthogonal signature can be labeled with $\log _{2} N$ bits, the feedback required, i.e., the label of each signature, is $\log _{2} N$ bits long. For the development of our signature assignment algorithm, we will assume that the orthogonality between the signatures is preserved.

When orthogonal temporal signatures are used, the optimization problems formulated in Section III reduce to a multiuser partitioning problem, i.e., finding subsets of users that will be assigned to each temporal signature. [25] shows that this problem is equivalent to finding a maximum independent set in a vertex weighted graph where each vertex represents a subset of the users. Specifically, in [25], finding the optimum partition with the highest average vertex weight is studied, where the weight of each vertex represents the sum capacity achieved by the corresponding subset of users. When the MSE is considered as the performance metric, the equivalence of the orthogonal signature assignment problem, and finding the maximum independent set in vertex weighted graph is still valid, with vertex weights defined as the MSEs achieved by the corresponding subset of users. In [26], weighted versions of finding the maximal independent set is shown to be NP-complete, and several approximation methods with polynomial complexity are presented in [27], [28]. Thus, for both performance metrics, the sum capacity and the MSE, the orthogonal temporal signature assignment problem is NP-complete. Fortunately, a low-complexity orthogonal temporal signature assignment algorithm that performs close to the performance bounds can be derived as explained next.

Recall that the optimum unconstrained effective signatures for $N<K \leq N N_{R}$ are those that are mutually orthogonal. However, the predefined spatial signatures of the users may prevent the effective signatures to be orthogonal to each other. Thus, we conclude that, a good heuristic is to try to assign the orthogonal temporal signatures to those users whose effective signatures, $\left\{\mathbf{A}_{j} \mathbf{s}_{j}\right\}$ 's, are as close to being orthogonal as possible. Observe that assigning more than $N_{R}$ users to the same temporal signature is likely to cause high correlation among the users. Besides the fact that each temporal signature should not be assigned to more than $N_{R}$ users, intuitively, it is logical to assign no more than $\lceil$ Number of users $/ N\rceil \leq N_{R}$ users to each temporal signature for the sake of fairness.

The above observations suggest that an $N$ step temporal signature assignment algorithm that tries to select the spatially less correlated users to 'share' each temporal signature is a good candidate for near-optimum performance. Specifically, at each step, the number of users that will be assigned for each temporal sig- nature is $\lceil$ Number of users $/ N\rceil$ which is guaranteed not to exceed $N_{R}$.

We now describe the proposed temporal signature assignment algorithm. The development assumes a fixed beamformer for each user. We will address the choice of beamformers in Section VI. Note that, at each orthogonal temporal signature assignment step, a performance penalty is incurred with respect to the performance bounds. Thus, intuitively, an effective temporal signature assignment strategy is to assign the user such that the least penalty is incurred. That is to say, we will aim for the smallest difference between the performance of the MIMOCDMA system and the prescribed performance bound.

The orthogonal temporal signature set is denoted by $\left\{\mathbf{e}_{i}\right\}_{i=1}^{N}$. When $\mathbf{e}_{i}$ is assigned to user $k$, its effective signature becomes $\mathbf{t}_{k}=\mathbf{A}_{k} \mathbf{e}_{i}$. Clearly, user $k$ interferes only with the users that employ the same the temporal signature $\mathbf{e}_{i}$.

\section{A. Sum Capacity}

When orthogonal temporal signatures are used, the sum capacity can be represented as

$$
C_{\text {sum }}=\sum_{i=1}^{N} C_{\mathcal{K}_{i}}=\sum_{i=1}^{N} \frac{1}{2} \log \left[\operatorname{det}\left(\mathbf{I}_{N_{R}}+\sigma^{-2} \sum_{j \in \mathcal{K}_{i}} \mathbf{a}_{j} \mathbf{a}_{j}^{H}\right)\right]
$$

where $\mathcal{K}_{i}$ is the set of users employing temporal signature $\mathbf{e}_{i}$, and $C_{\mathcal{K}_{i}}$ is the corresponding sum capacity. Assume that user $k$ is assigned $\mathbf{e}_{i}$. Define the set of users assigned to $\mathbf{e}_{i}$ excluding user $k$ as $\mathcal{K}_{i}-\{k\}=\overline{\mathcal{K}}_{i}^{(k)}$. The contribution of user $k$ to the sum capacity is simply

$$
\begin{aligned}
\Delta_{i k}^{\mathrm{sum}} & =C_{\mathcal{K}_{i}}-C_{\overline{\mathcal{K}}_{i}^{(k)}} \\
& =\frac{1}{2} \log \left[1+\mathbf{a}_{k}^{H}\left(\sigma^{2} \mathbf{I}_{N_{R}}+\sum_{j \in \overline{\mathcal{K}}_{i}^{(k)}} \mathbf{a}_{j} \mathbf{a}_{j}^{H}\right)^{-1} \mathbf{a}_{k}\right] .
\end{aligned}
$$

The capacity of user $k$ with its unconstrained effective signature, i.e., the single-user capacity is

$$
C_{k}=\frac{1}{2} \log \left(1+\sigma^{-2}\left\|\mathbf{a}_{k}\right\|^{2}\right) .
$$

Observe that, from user $k$ 's perspective, the assignment of user $k$ to $\mathbf{e}_{i}$ will result in a difference of $C_{k}-\Delta_{i k}^{\text {sum }}$ between the sum capacity upper bound and the achieved sum capacity. This difference can be expressed as

$$
\begin{aligned}
C_{k}- & \Delta_{i k}^{\text {sum }} \\
= & \frac{1}{2} \log \left[\frac{\left(1+\sigma^{-2}\left\|\mathbf{a}_{k}\right\|^{2}\right)}{\left(1+\mathbf{a}_{k}^{H}\left(\sigma^{2} \mathbf{I}_{N_{R}}+\sum_{j \in \overline{\mathcal{K}}_{i}^{(k)}} \mathbf{a}_{j} \mathbf{a}_{j}^{H}\right)^{-1} \mathbf{a}_{k}\right)}\right] .
\end{aligned}
$$

Thus, the user with the highest

$$
z_{i k}^{\text {sum }} \triangleq \frac{\left(1+\mathbf{a}_{k}^{H}\left(\sigma^{2} \mathbf{I}_{N_{R}}+\sum_{j \in \overline{\mathcal{K}}_{i}^{(k)}} \mathbf{a}_{j} \mathbf{a}_{j}^{H}\right)^{-1} \mathbf{a}_{k}\right)}{\left(1+\sigma^{-2}\left\|\mathbf{a}_{k}\right\|^{2}\right)}
$$


will result in the smallest difference from the upper bound from that user's perspective.

\section{B. Systemwide MSE}

A similar approach can be developed for the MSE. When orthogonal temporal signatures are used, the MSE can be represented as

MSE

$$
\begin{aligned}
& =\sum_{i=1}^{N} \operatorname{MSE}_{\mathcal{K}_{i}} \\
& =\sum_{i=1}^{N}\left[n\left(\mathcal{K}_{i}\right)-N_{R}+\sigma^{2} \operatorname{tr}\left\{\left(\sigma^{2} \mathbf{I}_{N_{R}}+\sum_{j \in \mathcal{K}_{i}} \mathbf{a}_{j} \mathbf{a}_{j}^{H}\right)^{-1}\right\}\right]
\end{aligned}
$$

where $\mathrm{MSE}_{\mathcal{K}_{i}}$ and $n\left(\mathcal{K}_{i}\right)$ are the total MSE and the cardinality of the user set $\mathcal{K}_{i}$, respectively. The contribution of user $k$ on the MSE is simply

$$
\begin{aligned}
& \Delta_{i k}^{\mathrm{MSE}}=\mathrm{MSE}_{\mathcal{K}_{i}}-\mathrm{MSE}_{\overline{\mathcal{K}}_{i}^{(k)}} \\
&=1-\sigma^{2} \frac{\mathbf{a}_{k}^{H}\left(\sigma^{2} \mathbf{I}_{N_{R}}+\sum_{j \in \overline{\mathcal{K}}_{i}^{(k)}} \mathbf{a}_{j} \mathbf{a}_{j}^{H}\right)^{-2} \mathbf{a}_{k}}{1+\mathbf{a}_{k}^{H}\left(\sigma^{2} \mathbf{I}_{N_{R}}+\sum_{j \in \overline{\mathcal{K}}_{i}^{(k)}} \mathbf{a}_{j} \mathbf{a}_{j}^{H}\right)^{-1} \mathbf{a}_{k}} .
\end{aligned}
$$

The MSE of the user $k$ with unconstrained effective signature orthogonal to all other users is

$$
\mathrm{MSE}_{k}=\frac{\sigma^{2}}{\sigma^{2}+\left\|\mathbf{a}_{k}\right\|^{2}}
$$

From user $k$ 's perspective, the assignment of user $k$ to $\mathbf{e}_{i}$ will result in a difference of $\Delta_{i k}^{\mathrm{MSE}}-\mathrm{MSE}_{k}$ between the MSE. Thus, the user with the highest

$$
z_{i k}^{\mathrm{MSE}} \triangleq \frac{1}{\Delta_{i k}^{\mathrm{MSE}}-\mathrm{MSE}_{k}}
$$

will result in the smallest difference from the lower bound from that user's perspective.

Consistent with the preceding discussion, we propose the following iterative algorithm. At each step, we choose the user that has the highest $z_{i k}^{\text {sum }}$ or $z_{i k}^{\mathrm{MSE}}$ to assign to $\mathbf{e}_{i}$ according to the performance metric chosen. This algorithm obviously favors the earlier temporal signatures, since these temporal signatures will have more users available to select from that will minimize the difference metric. However, by limiting the number of users using each orthogonal temporal signature by $\lceil$ Number of available users $/ N\rceil$ the earlier temporal signatures may have 1 user more than the later ones and the unfairness is somewhat diminished. An arbitrary user can be
TABLE II

ORTHOGONAL TEMPORAL SIGNATURE ASSIGNMENT

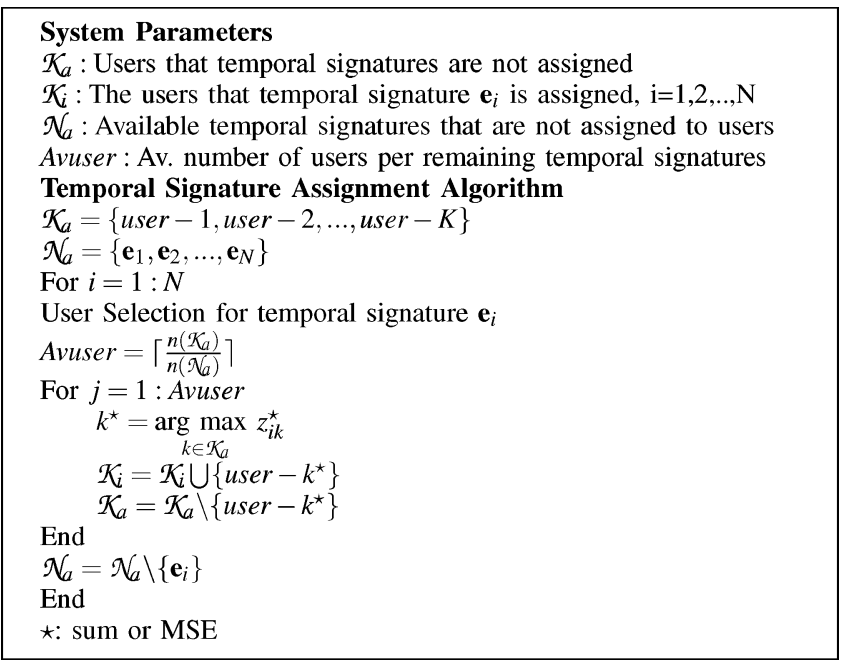

chosen from the available users as the first user to start the algorithm. The algorithm proposed is summarized in Table II, with $z_{i k}^{\mathrm{sum}}$ or $z_{i k}^{\mathrm{MSE}}$ substituted for $z_{i k}^{\star}$ for the desired performance metric, i.e., sum capacity or MSE. We note that the proposed algorithm assigns users to each signature, rather than assigning signatures to users. We run the algorithm sequentially over the users to be able to achieve efficient packing of spatially low correlated users to the same signature.

The orthogonal signature assignment algorithm derived so far is for single-path synchronous channels. For multipath and asynchronous channels, the asynchronous arrivals of users through multiple paths introduce additional interference. For this scenario, the interference from the users assigned to other orthogonal signatures should also be considered as well when assigning the temporal signatures to users. Using the same approach for orthogonal signature assignment algorithm and multipath and asynchronous model in (2)-(3), assigning user $k$ to signature $\mathbf{e}_{i}$ results in a gap of

$$
C_{k}-\Delta_{i k}^{\text {sum }}=\frac{1}{2} \log \frac{\left(1+\sigma^{-2} P_{\text {rec. } k}\right)}{\left(1+\mathbf{t}_{k}^{H}\left(\sigma^{2} \mathbf{I}_{\widetilde{N} N_{R}}+\sum_{j \in \overline{\mathcal{K}}^{(k)}} \mathbf{t}_{j} \mathbf{t}_{j}^{H}\right)^{-1} \mathbf{t}_{k}\right)}
$$

for the sum capacity, and

$$
\begin{aligned}
& \Delta_{i k}^{\mathrm{MSE}}-\mathrm{MSE}_{k} \\
& =1-\sigma^{2} \frac{\mathbf{t}_{k}^{H}\left(\sigma^{2} \mathbf{I}_{\widetilde{N} N_{R}}+\sum_{j \in \overline{\mathcal{K}}^{(k)}} \mathbf{t}_{j} \mathbf{t}_{j}^{H}\right)^{-2} \mathbf{t}_{k}}{1+\mathbf{t}_{k}^{H}\left(\sigma^{2} \mathbf{I}_{\widetilde{N}_{N}}+\sum_{j \in \overline{\mathcal{K}}^{(k)}} \mathbf{t}_{j} \mathbf{t}_{j}^{H}\right)^{-1} \mathbf{t}_{k}}-\frac{\sigma^{2}}{\sigma^{2}+P_{\text {rec. } k}}
\end{aligned}
$$

for the MSE. Once again, $\mathbf{t}_{k}=\mathbf{A}_{k} \mathbf{e}_{i}$, and $\overline{\mathcal{K}}^{(k)}$ is the set of users that are assigned a temporal signature except user $k$. Thus, 
corresponding decision metrics for multipath and asynchronous channels become

$$
\begin{aligned}
& z_{i k}^{\text {sum }} \triangleq \frac{\left(1+\mathbf{t}_{k}^{H}\left(\sigma^{2} \mathbf{I}_{\tilde{N} N_{R}}+\sum_{j \in \overline{\mathcal{K}}^{(k)}} \mathbf{t}_{j} \mathbf{t}_{j}^{H}\right)^{-1} \mathbf{t}_{k}\right)}{\left(1+\sigma^{-2} P_{\text {rec. } k}\right)} \\
& z_{i k}^{\mathrm{MSE}} \triangleq\left(1-\sigma^{2} \frac{\mathbf{t}_{k}^{H}\left(\sigma^{2} \mathbf{I}_{\widetilde{N} N_{R}}+\sum_{j \in \overline{\mathcal{K}}^{(k)}} \mathbf{t}_{j} \mathbf{t}_{j}^{H}\right)^{-2} \mathbf{t}_{k}}{1+\mathbf{t}_{k}^{H}\left(\sigma^{2} \mathbf{I}_{\tilde{N} N_{R}}+\sum_{j \in \overline{\mathcal{K}}^{(k)}} \mathbf{t}_{j} \mathbf{t}_{j}^{H}\right)^{-1} \mathbf{t}_{k}}\right. \\
& \left.-\frac{\sigma^{2}}{\sigma^{2}+P_{\text {rec. } k}}\right)^{-1} \text {. }
\end{aligned}
$$

Using the modified decision metrics (38) and (39) in Table II, the orthogonal signature assignment algorithm can be used for multipath and asynchronous MIMO-CDMA systems.

\section{BEAMFormer Design With ORTHOGONAL TEMPORAL SignATURES}

The previous section considered the case where transmit beamforming vectors are fixed. The performance of the MIMO-CDMA system is clearly a function of the choice of the transmit beamformers. In turn, the choice of the transmit beamformers is dependent on the available feedback level at the transmitter side. In this section, we consider different levels of feedback at the transmitter side, and determine the corresponding transmit beamformers to be employed. Specifically, the perfect transmit beamforming feedback case, the individual CSI feedback case where each user has only its own CSI and can beamform through its eigenmodes, and the antenna selection feedback case where only the index of the transmitter antenna is fed back, are investigated next.

The orthogonal temporal signature assignment algorithm in Table II easily accommodates the beamformer design in accordance with the feedback requirement, by an appropriate definition of the effective signature, as explained in the following.

\section{A. Perfect Transmit Beamformer Feedback}

In this section, we assume that the transmit beamformers can be designed without any feedback constraints. The feedback related to the temporal signature assignment is still limited to one of the $N$ labels of the orthogonal signatures. Note that, this is in contrast to the scenario in Section IV, where both signature and beamformer feedback were assumed to be unconstrained. The motivation for this section is to obtain a performance benchmark for the beamformer design with limited feedback.

For the set of effective spatial signatures, $\left\{\mathbf{a}_{j}=\right.$ $\left.\sqrt{P_{j}} \mathbf{H}_{j} \mathbf{f}_{j}\right\}_{j=1}^{K}$, the contribution of the transmit beamforming vector of user $k$ on the sum capacity, and the MSE can be expressed as

$$
\begin{aligned}
& \Delta_{i k}^{\text {sum }} \\
& =\frac{1}{2} \log \left[1+P_{k} \mathbf{f}_{k}^{H} \mathbf{H}_{k}^{H}\left(\sigma^{2} \mathbf{I}_{N_{R}}+\sum_{j \in \overline{\mathcal{K}}_{i}^{(k)}} \mathbf{a}_{j} \mathbf{a}_{j}^{H}\right)^{-1} \mathbf{H}_{k} \mathbf{f}_{k}\right] \\
& \Delta_{i k}^{\mathrm{MSE}} \\
& =1-\sigma^{2} \frac{P_{k} \mathbf{f}_{k}^{H} \mathbf{H}_{k}^{H}\left(\sigma^{2} \mathbf{I}_{N_{R}}+\sum_{j \in \overline{\mathcal{K}}_{i}^{(k)}} \mathbf{a}_{j} \mathbf{a}_{j}^{H}\right)^{-2} \mathbf{H}_{k} \mathbf{f}_{k}}{1+P_{k} \mathbf{f}_{k}^{H} \mathbf{H}_{k}^{H}\left(\sigma^{2} \mathbf{I}_{N_{R}}+\sum_{j \in \overline{\mathcal{K}}_{i}^{(k)}} \mathbf{a}_{j} \mathbf{a}_{j}^{H}\right)^{-1} \mathbf{H}_{k} \mathbf{f}_{k}} .
\end{aligned}
$$

When transmit beamformers can be optimized, the performance bounds for the sum capacity and MSE can be reformulated as

$$
\begin{aligned}
C_{k} & =\frac{1}{2} \log \left(1+\sigma^{-2} P_{k} \max _{\left\{\mathbf{f} \mid \mathbf{f}^{H} \mathbf{f}=1\right\}} \mathbf{f}^{H} \mathbf{H}_{k}^{H} \mathbf{H}_{k} \mathbf{f}\right) ; \\
\mathrm{MSE}_{k} & =\frac{\sigma^{2}}{\sigma^{2}+P_{k} \max _{\left\{\mathbf{f} \mid \mathbf{f}^{H} \mathbf{f}=1\right\}} \mathbf{f}^{H} \mathbf{H}_{k}^{H} \mathbf{H}_{k} \mathbf{f}} .
\end{aligned}
$$

This results in the following metrics, $z_{i k}^{\mathrm{sum}}$ and $z_{i k}^{\mathrm{MSE}}$

$$
\begin{aligned}
& z_{i k}^{\mathrm{sum}}\left(\mathbf{f}_{k}\right) \\
& =\frac{1+P_{k} \mathbf{f}_{k}^{H} \mathbf{H}_{k}^{H}\left(\mathbf{I}_{N_{R}}+\sigma^{-2} \sum_{j \in \overline{\mathcal{K}}_{i}^{(k)}} \mathbf{a}_{j} \mathbf{a}_{j}^{H}\right)^{-1} \mathbf{H}_{k} \mathbf{f}_{k}}{1+\sigma^{-2} P_{k} \max _{\left\{\mathbf{f} \mid \mathbf{f}{ }^{H}=1\right\}} \mathbf{f}^{H} \mathbf{H}_{k}^{H} \mathbf{H}_{k} \mathbf{f}} \\
& z_{i k}^{\mathrm{MSE}}\left(\mathbf{f}_{k}\right) \\
& =\left(1-\sigma^{2} \frac{P_{k} \mathbf{f}_{k}^{H} \mathbf{H}_{k}^{H}\left(\sigma^{2} \mathbf{I}_{N_{R}}+\sum_{j \in \overline{\mathcal{K}}_{i}^{(k)}} \mathbf{a}_{j} \mathbf{a}_{j}^{H}\right)^{-2} \mathbf{H}_{k} \mathbf{f}_{k}}{1+P_{k} \mathbf{f}_{k}^{H} \mathbf{H}_{k}^{H}\left(\sigma^{2} \mathbf{I}_{N_{R}}+\sum_{j \in \overline{\mathcal{K}}_{i}^{(k)}} \mathbf{a}_{j} \mathbf{a}_{j}^{H}\right)^{-1} \mathbf{H}_{k} \mathbf{f}_{k}}\right. \\
& \left.-\frac{\sigma^{2}}{\sigma^{2}+P_{k} \max _{\left\{\mathbf{f} \mid \mathbf{f}^{H} \mathbf{f}=1\right\}} \mathbf{f}^{H} \mathbf{H}_{k}^{H} \mathbf{H}_{k} \mathbf{f}}\right)
\end{aligned}
$$

respectively. We observe that the transmit beamforming vector that maximizes $z_{i k}^{\text {sum }}$ is the eigenvector which corresponds to the maximum eigenvalue of $\mathbf{H}_{k}^{H}\left(\sigma^{2} \mathbf{I}_{N_{R}}+\sum_{j \in \overline{\mathcal{K}}_{i}^{(k)}} \mathbf{a}_{j} \mathbf{a}_{j}^{H}\right)^{-1} \mathbf{H}_{k}$, and the transmit beamforming vector that maximizes $z_{i k}^{\mathrm{MSE}}$ is the generalized eigenvector which corresponds to the maximum generalized eigenvalue of $\mathbf{H}_{k}^{H}\left(\sigma^{2} \mathbf{I}_{N_{R}}+\sum_{j \in \overline{\mathcal{K}}_{i}^{(k)}} \mathbf{a}_{j} \mathbf{a}_{j}^{H}\right)^{-2} \mathbf{H}_{k}$ and $\left(1 / P_{k}\right) \mathbf{I}+\mathbf{H}_{k}^{H}\left(\sigma^{2} \mathbf{I}_{N_{R}}+\sum_{j \in \overline{\mathcal{K}}_{i}^{(k)}} \mathbf{a}_{j} \mathbf{a}_{j}^{H}\right)^{-1} \mathbf{H}_{k}$. 
For multipath asynchronous channels, the contribution of the users, the performance bounds, and thus, the decision metrics $z_{i k}^{\text {sum }}$ and $z_{i k}^{\mathrm{MSE}}$, are functions of both the transmit beamformer vectors and the temporal signatures. The performance bounds are

$$
\begin{aligned}
C_{k} & =\frac{1}{2} \log \left(1+\sigma^{-2} \max _{\left\{\mathbf{f} \mid \mathbf{f}^{H} \mathbf{f}=1\right\}} \mathbf{f}^{H} \mathbf{Q}_{k}^{H} \mathbf{Q}_{k} \mathbf{f}\right) ; \\
\mathrm{MSE}_{k} & =\frac{\sigma^{2}}{\sigma^{2}+\max _{\left\{\mathbf{f} \mid \mathbf{f}^{H} \mathbf{f}=1\right\}} \mathbf{f}^{H} \mathbf{Q}_{k}^{H} \mathbf{Q}_{k} \mathbf{f}}
\end{aligned}
$$

and $\mathbf{Q}_{k}$ in (3) is obtained using signature $\mathbf{e}_{i}$. Thus, we have

$$
\begin{aligned}
& z_{i k}^{\text {sum }}\left(\mathbf{f}_{k}\right) \\
& =\frac{1+\mathbf{f}_{k}^{H} \mathbf{Q}_{k}^{H}\left(\mathbf{I}_{\widetilde{N} N_{R}}+\sigma^{-2} \sum_{j \in \overline{\mathcal{K}}^{(k)}} \mathbf{t}_{j} \mathbf{t}_{j}^{H}\right)^{-1} \mathbf{Q}_{k} \mathbf{f}_{k}}{1+\sigma^{-2} \max _{\left\{\mathbf{f} \mid \mathbf{f}^{H} \mathbf{f}=1\right\}} \mathbf{f}^{H} \mathbf{Q}_{k}^{H} \mathbf{Q}_{k} \mathbf{f}} \\
& z_{i k}^{\mathrm{MSE}}\left(\mathbf{f}_{k}\right) \\
& =\left(1-\sigma^{2} \frac{\mathbf{f}_{k}^{H} \mathbf{Q}_{k}^{H}\left(\sigma^{2} \mathbf{I}_{\widetilde{N} N_{R}}+\sum_{j \in \overline{\mathcal{K}}^{(k)}} \mathbf{t}_{j} \mathbf{t}_{j}^{H}\right)^{-2} \mathbf{Q}_{k} \mathbf{f}_{k}}{1+\mathbf{f}_{k}^{H} \mathbf{Q}_{k}^{H}\left(\sigma^{2} \mathbf{I}_{\widetilde{N} N_{R}}+\sum_{j \in \overline{\mathcal{K}}^{(k)}} \mathbf{t}_{j} \mathbf{t}_{j}^{H}\right)^{-1} \mathbf{Q}_{k} \mathbf{f}_{k}}\right. \\
& \left.-\frac{\sigma^{2}}{\sigma^{2}+\max _{\left\{\mathbf{f} \mid \mathbf{f} \mathbf{f}^{H} \mathbf{f}=1\right\}} \mathbf{f}^{H} \mathbf{Q}_{k}^{H} \mathbf{Q}_{k} \mathbf{f}}\right)^{-1} \text {. }
\end{aligned}
$$

For multipath asynchronous channels, the transmit beamforming vector that maximizes $z_{i k}^{\text {sum }}$ is the eigenvector which corresponds to the maximum eigenvalue of $\mathbf{Q}_{k}^{H}\left(\sigma^{2} \mathbf{I}_{\widetilde{N} N_{R}}+\right.$ $\left.\sum_{j \in \overline{\mathcal{K}}^{(k)}} \mathbf{t}_{j} \mathbf{t}_{j}^{H}\right)^{-1} \mathbf{Q}_{k}$, and the transmit beamforming vector that maximizes $z_{i k}^{\mathrm{MSE}}$ is the generalized eigenvector which corresponds to the maximum generalized eigenvalue of $\mathbf{Q}_{k}^{H}\left(\sigma^{2} \mathbf{I}_{\widetilde{N}_{N}}+\sum_{j \in \overline{\mathcal{K}}^{(k)}} \mathbf{t}_{j} \mathbf{t}_{j}^{H}\right)^{-2} \mathbf{Q}_{k}$ and $\mathbf{I}+\mathbf{Q}_{k}^{H}\left(\sigma^{2} \mathbf{I}_{\widetilde{N} N_{R}}+\sum_{j \in \overline{\mathcal{K}}(k)} \mathbf{t}_{j} \mathbf{t}_{j}^{H}\right)^{-1} \mathbf{Q}_{k}$.

The orthogonal temporal signature assignment algorithm with perfect transmit beamforming feedback (PTBF) should compare the performance of the users with their best transmit beamforming vectors at each step for both single and multipath channels. The resulting signature assignment and beamformer design algorithm is a two-step algorithm. The best transmit beamformers are found at the first step, and the user with the highest $z_{i k}^{\star}$, i.e., $z_{i k}^{\mathrm{sum}}$ or $z_{i k}^{\mathrm{MSE}}$, is assigned a temporal signature next. The orthogonal temporal signature assignment algorithm with perfect transmit beamforming feedback is the algorithm presented in Table II with the selection criterion defined as

$$
z_{i k}^{\star}=\max _{\left|\mathbf{f}_{k}\right|=1} z_{i k}^{\star}\left(\mathbf{f}_{k}\right)
$$

After the assignment of the temporal signatures, the users may choose to update their transmit beamformers improving the performance further at each step. In the numerical results, we have chosen to perform a single iteration in order to provide a fair comparison with the limited feedback designs given in the next two sections, i.e., eigenmode and antenna selection.

\section{B. Individual CSI Feedback}

In the case of limited CSI, a feasible scenario is that each user knows its own channel; for example in a time division duplex system. The transmit beamforming vector of each user can be chosen among the set of eigenvectors of the channel matrices, $\left\{\mathbf{H}_{k}^{H} \mathbf{H}_{k}\right\}$, i.e., $\left\{\mathbf{u}_{k m}\right\}_{m=1}^{N_{T}}$, and the only required feedback between the user and the receiver is which eigenmodes should be used. Consider the case where one eigenmode is selected. When user $k$ selects eigenmode $m, \mathbf{a}_{k}=\sqrt{P_{k}} \mathbf{H}_{k} \mathbf{u}_{k m}$ where $\mathbf{u}_{k m}$ is the $m$ th eigenvector of $\mathbf{H}_{k}^{H} \mathbf{H}_{k}$. It is clear that the received power of the user $k,\left\|\mathbf{a}_{k}\right\|^{2}=P_{k} \mathbf{u}_{k m}^{H} \mathbf{H}_{k}^{H} \mathbf{H}_{k} \mathbf{u}_{k m}$, is maximized when the eigenvector corresponding to the maximum eigenvalue is used. In this case, the single-user performance bounds, $C_{k}$ and $\mathrm{MSE}_{k}$ for the eigenmode selection remains the same as in perfect transmit beamforming case, i.e., (41).

Observe from (42) and (43) that, the metrics are both dependent on the received power and the effective spatial signatures of the users that are already assigned. Assuming that we will assign users with spatially low correlations to the same orthogonal temporal signature, intuitively, an effective method is to choose the eigenmode that will maximize the received power of the user, i.e., the eigenmode which corresponds to the maximum eigenvalue. In this case, the transmit beamformer and the effective spatial signature of user $k$ become

$$
\mathbf{f}_{k}=\underset{\mathbf{u}_{k m} \mid m \in\left\{1,2, \ldots, N_{T}\right\}}{\arg \max } \mathbf{u}_{k m}^{H} \mathbf{H}_{k}^{H} \mathbf{H}_{k} \mathbf{u}_{k m} ; \mathbf{a}_{k}=\sqrt{P_{k}} \mathbf{H}_{k} \mathbf{f}_{k} .
$$

The orthogonal temporal signature assignment algorithm with maximum received power eigenmode selection (MES) is the algorithm presented in Table II with the spatial signatures defined as the spatial signatures with the eigenmodes which correspond to the maximum eigenvalues, i.e., as in (48).

MES requires no feedback to the transmitter side related to beamforming when time division duplex mode is employed. It is expected to perform well especially if one eigenvalue is significantly larger than the others, and the MIMO channels of the users are independent of each other. On the other hand, if the channels of the users are highly correlated, choosing the eigenmode that maximizes the received power may not significantly outperform the remaining eigenmodes. In that case, we may choose to employ an orthogonal temporal signature assignment algorithm that considers each eigenmode, and chooses the eigenmode of the user that has the best performance in the sense of minimizing the performance difference from a single user's perspective. We term this algorithm the orthogonal temporal signature assignment algorithm with generalized eigenmode selection (GES).

The algorithm with generalized eigenmode selection is a two-step algorithm where the best eigenmodes are found at the first step, and the user with the highest $z_{i k}^{\mathrm{sum}}$ or $z_{i k}^{\mathrm{MSE}}$ is assigned a temporal signature next. We have (49) and (50), 
shown at the bottom of the page, where $\lambda_{k, m}$ is the $m$ th eigenvalue of $\mathbf{H}_{k}^{H} \mathbf{H}_{k}$. The effective spatial signature of user $k$ is $\mathbf{a}_{k}=\sqrt{P_{k}} \mathbf{H}_{k} \mathbf{u}_{k, \bar{m}}$ when $\bar{m}$ th eigenmode is selected.

The extension of MES and GES for multipath and asynchronous channels requires the users to have their individual CSI for each path, i.e., $\mathbf{H}_{k, 1}, \mathbf{H}_{k, 2}, \ldots, \mathbf{H}_{k, \widetilde{L}}$ for user $k$. Using this information together with the candidate orthogonal temporal signature, $\mathbf{e}_{i}$, each user has their individual $\mathbf{Q}_{k}$ and may select one of the eigenvectors of $\mathbf{Q}_{k}^{H} \mathbf{Q}_{k}$, i.e., $\left\{\mathbf{v}_{k m}\right\}_{m=1}^{N_{T}}$, as their transmit beamforming vector. The MES algorithm for multipath asynchronous channels thus chooses the transmit beamformer as

$$
\mathbf{f}_{k}=\underset{\mathbf{v}_{k m} \mid m \in\left\{1,2, \ldots, N_{T}\right\}}{\arg \max } \mathbf{v}_{k m}^{H} \mathbf{Q}_{k}^{H} \mathbf{Q}_{k} \mathbf{v}_{k m}
$$

MES for multipath asynchronous channels is the algorithm presented in Table II with the transmit beamformers of users defined as in (51). Similarly, GES for multipath asynchronous channels should compare each eigenmode and choose the best eigenmode in the sense of minimizing the gap between the achieved performance and performance bound. The decision metrics for multipath asynchronous channels become (52) and (53), shown at the bottom of the page. GES for multipath asynchronous channels is the algorithm presented in Table II with these decision metrics.

\section{Antenna Selection Feedback}

An alternative approach for limited feedback is antenna selection, where the only required feedback is which antenna(s) should be used [29], [30]. Consider the case where one transmitter antenna is selected. In this case, the spatial signature of user $k$ is that of the selected transmitter antenna, $m$, i.e., $\mathbf{a}_{k}=\sqrt{P_{k}} \mathbf{h}_{k m}$, where $\mathbf{h}_{k m}$ is the $m$ th column vector of user $k$ 's channel matrix, and the received power of user $k$ is $\left\|\mathbf{a}_{k}\right\|^{2}=$ $P_{k}\left\|\mathbf{h}_{k, m}\right\|^{2}$. Following the same approach as in the eigenmode selection, intuitively, an effective antenna selection method is to choose the transmitter antenna that will maximize the received power of the user, i.e., the transmitter antenna with the highest norm $\left\|\mathbf{h}_{k m}\right\|$. In this case, the spatial signature of the user is

$$
\bar{m}=\underset{m \in\left\{1,2, \ldots, N_{T}\right\}}{\arg \max }\left\|\mathbf{h}_{k, m}\right\| \quad \mathbf{a}_{k}=\sqrt{P_{k}} \mathbf{h}_{k, \bar{m}}
$$

The orthogonal temporal signature assignment algorithm with maximum received power antenna selection (MAS) is the algorithm presented in Table II with the effective spatial

$$
\begin{aligned}
& z_{i k}^{\text {sum }}=\max _{m}\left(\frac{\left.1+P_{k} \mathbf{u}_{k, m}^{H} \mathbf{H}_{k}^{H}\left(\sigma^{2} \mathbf{I}_{N_{R}}+\sum_{j \in \overline{\mathcal{K}}_{i}^{(k)}} \mathbf{a}_{j} \mathbf{a}_{j}^{H}\right)^{-1} \mathbf{H}_{k} \mathbf{u}_{k, m}\right)}{1+\sigma^{-2} P_{k} \max _{m^{\prime}} \lambda_{k, m^{\prime}}}\right) \\
& z_{i k}^{\mathrm{MSE}}=\max _{m}\left(1-\frac{\sigma^{2} P_{k} \mathbf{u}_{k, m}^{H} \mathbf{H}_{k}^{H}\left(\sigma^{2} \mathbf{I}_{N_{R}}+\sum_{j \in \overline{\mathcal{K}}_{i}^{(k)}} \mathbf{a}_{j} \mathbf{a}_{j}^{H}\right)^{-2} \mathbf{H}_{k} \mathbf{u}_{k, m}}{1+P_{k} \mathbf{u}_{k, m}^{H} \mathbf{H}_{k}^{H}\left(\sigma^{2} \mathbf{I}_{N_{R}}+\sum_{j \in \overline{\mathcal{K}}_{i}^{(k)}} \mathbf{a}_{j} \mathbf{a}_{j}^{H}\right)^{-1} \mathbf{H}_{k} \mathbf{u}_{k, m}}-\frac{\sigma^{2}}{\sigma^{2}+P_{k} \max _{m^{\prime}} \lambda_{k, m^{\prime}}}\right)^{-1} .
\end{aligned}
$$

$$
\begin{aligned}
z_{i k}^{\text {sum }} & =\max _{m}\left(\frac{\left.1+\mathbf{v}_{k, m}^{H} \mathbf{Q}_{k}^{H}\left(\sigma^{2} \mathbf{I}_{\widetilde{N} N_{R}}+\sum_{j \in \overline{\mathcal{K}}^{(k)}} \mathbf{t}_{j} \mathbf{t}_{j}^{H}\right)^{-1} \mathbf{Q}_{k} \mathbf{v}_{k, m}\right)}{1+\sigma^{-2} \max _{m^{\prime}} \mathbf{v}_{k, m^{\prime}}^{H} \mathbf{Q}_{k}^{H} \mathbf{Q}_{k} \mathbf{v}_{k, m^{\prime}}}\right) \\
z_{i k}^{\mathrm{MSE}} & =\max _{m}\left(1-\frac{\sigma^{2} \mathbf{v}_{k, m}^{H} \mathbf{Q}_{k}^{H}\left(\sigma^{2} \mathbf{I}_{\widetilde{N} N_{R}}+\sum_{j \in \overline{\mathcal{K}}^{(k)}} \mathbf{t}_{j} \mathbf{t}_{j}^{H}\right)^{-2} \mathbf{Q}_{k} \mathbf{v}_{k, m}}{1+\mathbf{v}_{k, m}^{H} \mathbf{Q}_{k}^{H}\left(\sigma^{2} \mathbf{I}_{\widetilde{N} N_{R}}+\sum_{j \in \overline{\mathcal{K}}^{(k)}} \mathbf{t}_{j} \mathbf{t}_{j}^{H}\right)^{-1} \mathbf{Q}_{k} \mathbf{v}_{k, m}}-\frac{\sigma^{2}}{\sigma^{2}+\max _{m^{\prime}} \mathbf{v}_{k, m^{\prime}}^{H} \mathbf{Q}_{k}^{H} \mathbf{Q}_{k} \mathbf{v}_{k, m^{\prime}}}\right)^{-1} .
\end{aligned}
$$


signatures defined as the spatial signatures of the transmitter antennas with the highest norm.

This approach is expected to perform well if one transmitter antenna has a spatial signature whose norm is significantly larger than the others, and the MIMO channels of the users are independent of each other. If this is not the case, a similar approach to the generalized eigenmode selection that compares the performances of all transmitter antennas in terms of minimizing the performance difference from a single user's perspective, can be devised. We term this algorithm the orthogonal temporal signature assignment algorithm with generalized antenna selection (GAS). Note that the performance bounds that are defined by the maximum received power of the users become

$$
\begin{aligned}
C_{k} & =\frac{1}{2} \log \left(1+\sigma^{-2} P_{k} \max _{m=1, \ldots, N_{T_{k}}}\left\|\mathbf{h}_{k, m}\right\|^{2}\right) ; \\
\mathrm{MSE}_{k} & =\frac{\sigma^{2}}{\sigma^{2}+P_{k} \max _{m=1, \ldots, N_{T_{k}}}\left\|\mathbf{h}_{k, m}\right\|^{2}}
\end{aligned}
$$

for the sum capacity and the MSE, respectively, and they are different than the performance bounds in (41). Using the same approach in GES and the performance bounds (55), we have
(56) and (57), shown at the bottom of the page. The resulting signature assignment and antenna selection algorithm is a twostep algorithm where the best transmit antennas are found at the first step, and the user with the highest $z_{i k}^{\mathrm{sum}}$ or $z_{i k}^{\mathrm{MSE}}$ is assigned a temporal signature next.

For multipath asynchronous channels, the orthogonal temporal signature assignment algorithm with antenna selection should choose one of the transmitter antennas resulting the effective spatial temporal signature of user $k, \mathbf{t}_{k}$, to be a column of $\mathbf{Q}_{k}$, i.e., $\mathbf{t}_{k}=\tilde{\mathbf{h}}_{k, m}$ where $\mathbf{Q}_{k}=\left[\tilde{\mathbf{h}}_{k, 1}, \tilde{\mathbf{h}}_{k, 2}, \ldots, \tilde{\mathbf{h}}_{k, N_{T}}\right]$. Thus, MAS algorithm for multipath asynchronous channels chooses the best transmitter antenna as

$$
\bar{m}=\underset{m \in\left\{1,2, \ldots, N_{T}\right\}}{\arg \max }\left\|\tilde{\mathbf{h}}_{k, m}\right\| ; \quad \mathbf{t}_{k}=\tilde{\mathbf{h}}_{k, \bar{m}}
$$

and the decision metrics in GAS algorithm for multipath asynchronous channels become (59) and (60), shown at the bottom of the page.

In this section, we proposed several transmit beamformer design algorithms, namely, PFTB, MES, GES, MAS and GAS, combined with orthogonal temporal signature assignment considering the level of available feedback. The feedback requirements of the algorithms are summarized in Table III. In the nu-

$$
\begin{aligned}
z_{i k}^{\mathrm{sum}} & =\max _{m}\left(\frac{1+P_{k} \mathbf{h}_{k, m}^{H}\left(\sigma^{2} \mathbf{I}_{N_{R}}+\sum_{j \in \overline{\mathcal{K}}_{i}^{(k)}} \mathbf{a}_{j} \mathbf{a}_{j}^{H}\right)^{-1} \mathbf{h}_{k, m}}{1+\sigma^{-2} P_{k} \max _{m^{\prime}} \mathbf{h}_{k, m^{\prime}}^{H} \mathbf{h}_{k, m^{\prime}}}\right) \\
z_{i k}^{\mathrm{MSE}}=\max _{m} & \left(\begin{array}{l}
1-\sigma^{2} \frac{P_{k} \mathbf{h}_{k, m}^{H}\left(\sigma^{2} \mathbf{I}_{N_{R}}+\sum_{j \in \overline{\mathcal{K}}_{i}^{(k)}} \mathbf{a}_{j} \mathbf{a}_{j}^{H}\right)^{-2} \mathbf{h}_{k, m}}{1+P_{k} \mathbf{h}_{k, m}^{H}\left(\sigma^{2} \mathbf{I}_{N_{R}}+\sum_{j \in \overline{\mathcal{K}}_{i}^{(k)}} \mathbf{a}_{j} \mathbf{a}_{j}^{H}\right)^{-1}}-\frac{\sigma^{2}}{\mathbf{h}_{k, m}^{2}+P_{k} \max _{m^{\prime}}\left\|\mathbf{h}_{k, m^{\prime}}\right\|^{2}}
\end{array}\right)^{-1} .
\end{aligned}
$$

$$
\begin{aligned}
z_{i k}^{\mathrm{sum}} & =\max _{m}\left(\frac{\left.1+\tilde{\mathbf{h}}_{k, m}^{H}\left(\sigma^{2} \mathbf{I}_{\tilde{N} N_{R}}+\sum_{j \in \overline{\mathcal{K}}^{(k)}} \mathbf{t}_{j} \mathbf{t}_{j}^{H}\right)^{-1} \tilde{\mathbf{h}}_{k, m}\right)}{1+\sigma^{-2} \max _{m^{\prime}} \tilde{\mathbf{h}}_{k, m^{\prime}}^{H} \tilde{\mathbf{h}}_{k, m^{\prime}}}\right) \\
z_{i k}^{\mathrm{MSE}} & =\max _{m}\left(1-\sigma^{2} \frac{\tilde{\mathbf{h}}_{k, m}^{H}\left(\sigma^{2} \mathbf{I}_{\widetilde{N} N_{R}}+\sum_{j \in \overline{\mathcal{K}}^{(k)}} \mathbf{t}_{j} \mathbf{t}_{j}^{H}\right)^{-2} \tilde{\mathbf{h}}_{k, m}}{1+\tilde{\mathbf{h}}_{k, m}^{H}\left(\sigma^{2} \mathbf{I}_{\tilde{N} N_{R}}+\sum_{j \in \overline{\mathcal{K}}^{(k)}} \mathbf{t}_{j} \mathbf{t}_{j}^{H}\right)^{-1} \tilde{\mathbf{h}}_{k, m}}-\frac{\sigma^{2}}{\sigma^{2}+\max _{m^{\prime}}\left\|\tilde{\mathbf{h}}_{k, m^{\prime}}\right\|^{2}}\right)^{-1} .
\end{aligned}
$$


TABLE III

COMPARISON OF FEEDBACK REQUIREMENTS

\begin{tabular}{|l|c|c|c|}
\hline Scheme & Beamformer Feedback & Signature Feedback & Update Frequency \\
\hline \hline PF & Perfect & Perfect & 1 Update/user/iteration \\
\hline PTBF & Perfect & $\log _{2} N$ & 1 Update/user \\
\hline GES & Index of Eigen Mode & $\log _{2} N$ & 1 Update/user \\
\hline GAS & Index of Tx Antenna & $\log _{2} N$ & 1 Update/user \\
\hline MES & - & $\log _{2} N$ & 1 Update/user \\
\hline MAS & - & $\log _{2} N$ & 1 Update/user \\
\hline
\end{tabular}

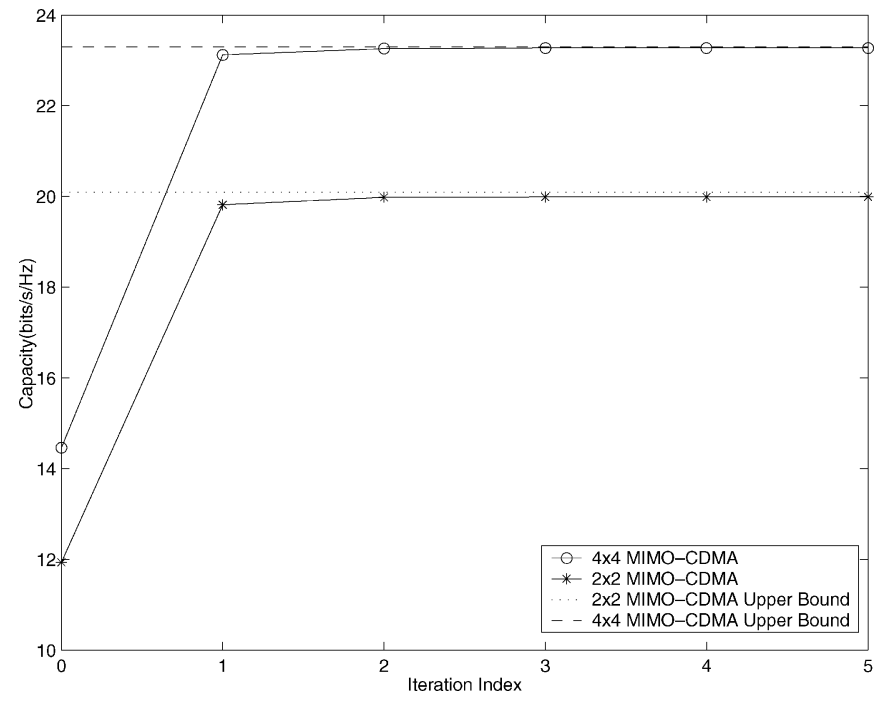

Fig. 2. $K=12$ user $2 \times 2$ and $4 \times 4$ MIMO-CDMA system.

merical results shown next, we will investigate the performance of the proposed methods.

\section{NUMERICAL RESUlTS AND DISCUSSION}

In this section, we present numerical results related to the performance of the signature and beamformer design algorithms. We compare the performance of the transmit strategies for different levels of feedback to investigate the benefit gained by exploiting the channel state information. For the sake of a fair comparison, we use the same performance upper bound for all scenarios. In particular, we use the upper bound when each user has the beamformer which is the eigenvector that corresponds to the maximum eigenvalue of its channel. We note that the performance of this beamformer provides an upper bound for all scenarios. The simulations are performed for $K=12$ user, $2 \times 2$ and $4 \times 4$ MIMO-CDMA systems with a processing gain of $N=8$. The channels are realizations of a flat fading channel model where all links are assumed to be independent and identically distributed complex Gaussian random variables. The received SNR of each user is $7 \mathrm{~dB}$. CDF curves for sum capacity and MSE obtained by simulating 10000 channel realizations are presented.

First, we consider $2 \times 2$ and $4 \times 4$ MIMO-CDMA systems with given channel realizations and investigate the performance of the iterative signature and beamformer design algorithm (PF) described in Table I. Figs. 2 and 3 show the evolution of the algorithms for the sum capacity and the MSE respectively. It is observed that the iterative algorithms proposed converge fast, and the first updates of the temporal signatures and beamformers

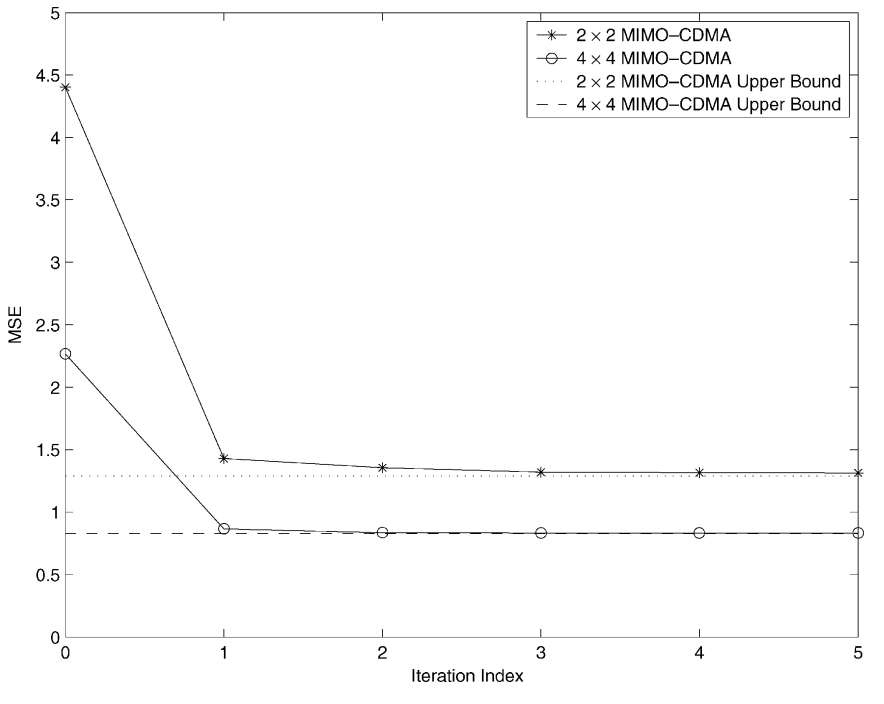

Fig. 3. $K=12$ user $2 \times 2$ and $4 \times 4$ MIMO-CDMA system.

provide substantial improvement in the performance metric considered, a trend we have observed throughout our simulations, with random starting points. Thus, one may choose to update the temporal signatures and beamformers only once for each user restricting the feedback requirement for each user to minimum. We have also observed that different random starting points converged to the same sum capacity/MSE though they may require different number of iterations to converge. We observe that the proposed iterative algorithms perform very close to the performance bounds. As expected, as the number of transmit/receive antennas is increased, better performance can be achieved since each added transmit/receive antenna provides additional spatial diversity.

We compare the performance of the signature and transmit beamformer design strategies for $K=12$ user $2 \times 2$ and $4 \times 4$ MIMO-CDMA system with different levels of feedback to investigate the benefit of exploiting the CSI in Figs. 4-9 in terms of the sum capacity and MSE. We also provide the performance of MIMO-CDMA systems, in which temporal and spatial transmitter optimization are done separately, to present the gain we obtain by jointly exploiting the spatial and temporal characteristics of the system: i) a system in which users employ the eigenvectors which correspond to the maximum eigenvalues of their channels as their beamformers, and are assigned Generalized Welch Bound Equality (WBE) signature sequences (Generalized WBE with ES), and ii) a system in which users transmit through the antenna that will maximize their received power, and are assigned Generalized Welch Bound Equality (WBE) signature sequences (Generalized WBE with AS). In Fig. 4, 


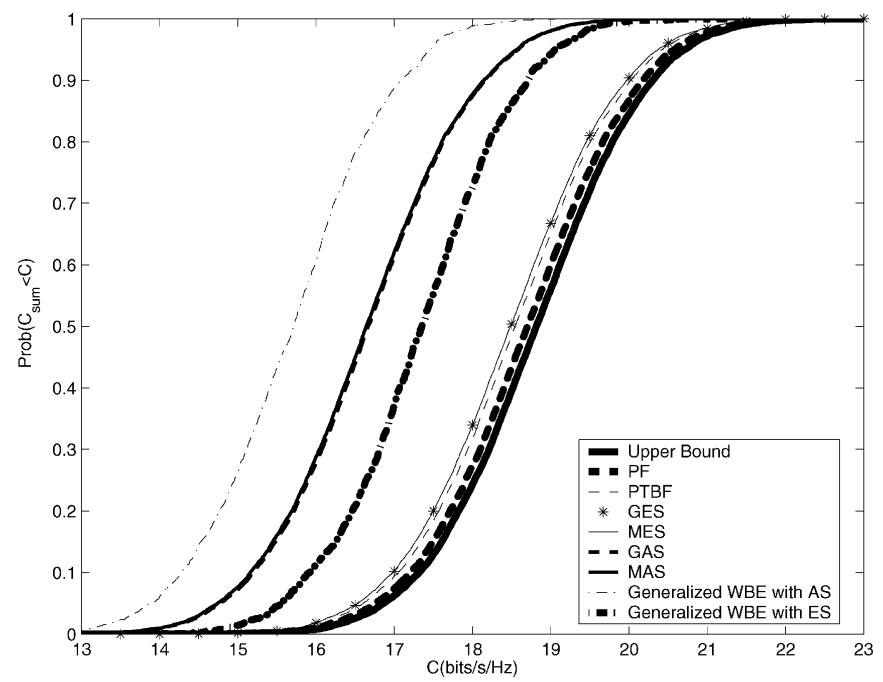

Fig. 4. $K=12$ user MIMO-CDMA system with $2 \times 2$ MIMO model and $N=8$. Comparison of the sum capacity CDF curves of the upper bound, the MIMO-CDMA systems with different levels of feedback and Generalized WBE sequences.

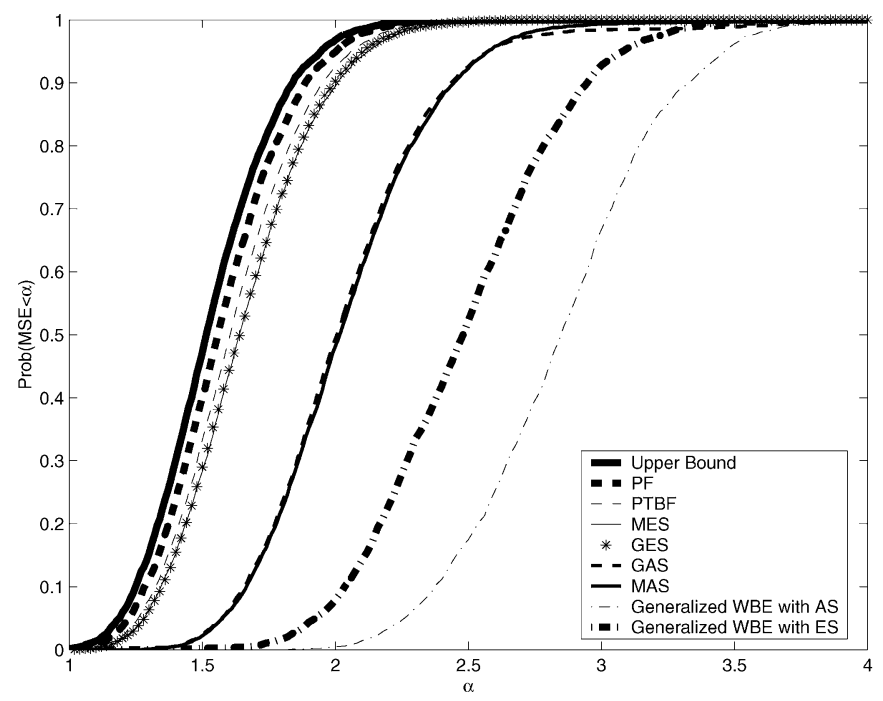

Fig. 5. $K=12$ user MIMO-CDMA system with $2 \times 2$ MIMO model and $N=8$. Comparison of the MSE CDF curves of the lower bound, the MIMO-CDMA systems with different levels of feedback and Generalized WBE sequences.

we present the sum capacity performances of all algorithms we proposed as well as the systems with WBE signatures for $2 \times 2$ MIMO-CDMA system. We observe that considering both spatial and temporal domains jointly, i.e., utilizing orthogonal signatures with appropriate beamformers performs better than the performance of the systems with WBE signatures and antenna selection or eigenbeamforming. The results in Fig. 5 show the same performance improvement over generalized WBE sequences for the MSE performance metric. We observe that the performance is improved as the level of feedback increases, and the signature and transmit beamformer design algorithm with iterative updates $(\mathrm{PF})$ performs the best. Notice that the generalized beamformer design approach that considers the performance of all transmitter antennas/eigenmodes with the orthogonal temporal signature assignment (GAS/GES) outperforms the maximum received power antennas/eigenmodes selection

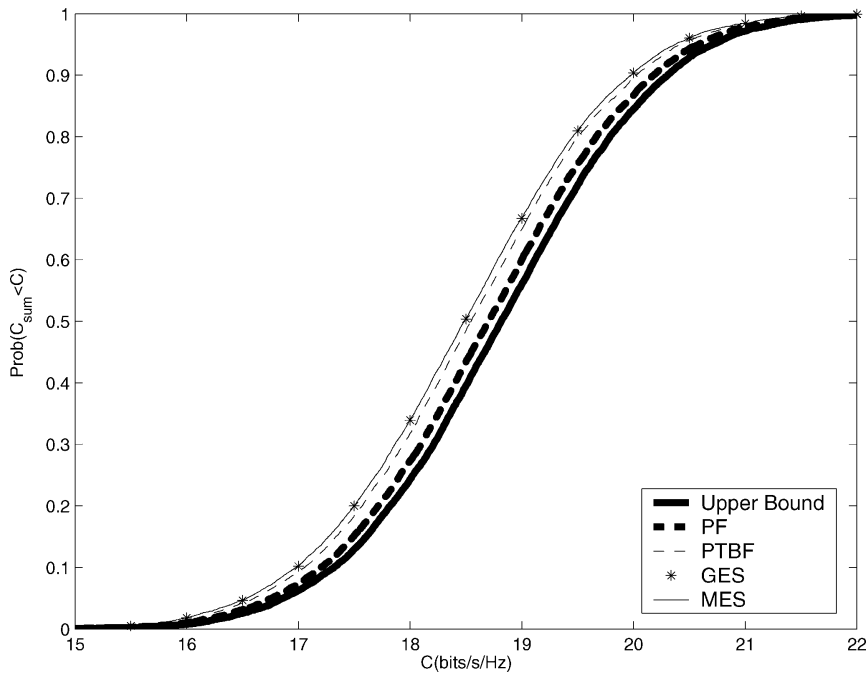

Fig. 6 . $K=12$ user MIMO-CDMA system with $2 \times 2$ MIMO model and $N=8$. Comparison of the sum capacity CDF curves of the upper bound and the MIMO-CDMA systems with different levels of feedback.

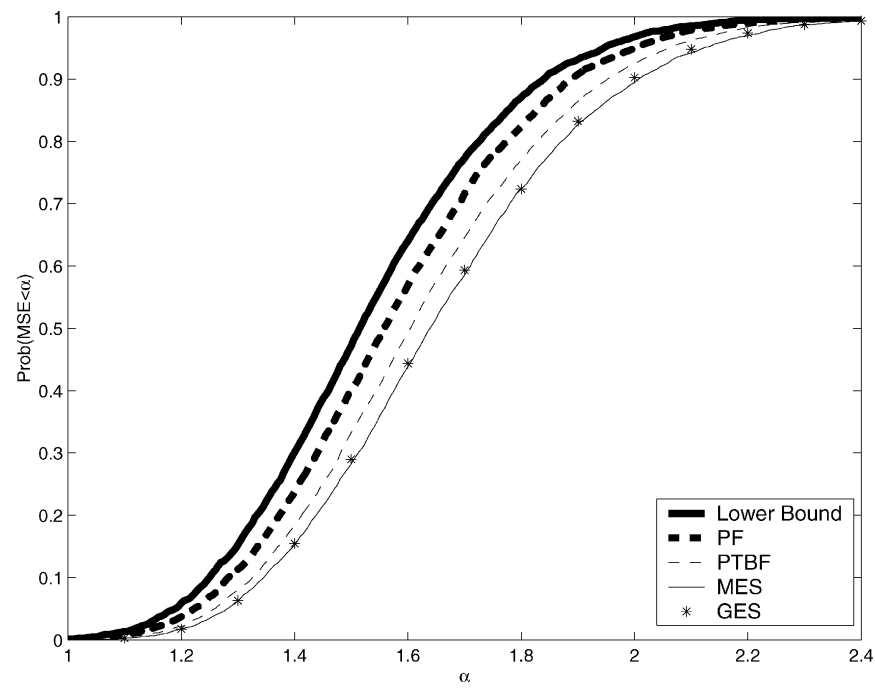

Fig. 7. $K=12$ user MIMO-CDMA system with $2 \times 2$ MIMO model and $N=8$. Comparison of the MSE CDF curves of the lower bound and the MIMO-CDMA systems with different levels of feedback.

approach (MAS/MES). However, the largest relative gain is due to the feedback related to individual CSI, i.e., the eigenmode selection methods, combined with the orthogonal temporal signature assignment algorithm. We observe in Figs. 4 and 5 that orthogonal temporal signature assignment with antenna selection, which does not consider the best (single or multiuser) eigenmodes to transmit, performs poorly with respect to the other algorithms we proposed. In Figs. 6 and 7, we observe that the performance of GES, GAS, PTBF and PF are very close to the described performance upper bounds, and hence the optimum performance in terms sum capacity and MSE, respectively. In Figs. 8 and 9, we also provide similar performance comparison for a $4 \times 4$ MIMO-CDMA system. Observe that for a given outage probability, the difference between the achieved sum capacity and the upper bound is no more than $0.5 \mathrm{bits} / \mathrm{s} / \mathrm{Hz}$ for $2 \times 2$ MIMO-CDMA system, and $0.25 \mathrm{bits} / \mathrm{s} / \mathrm{Hz}$ for $4 \times 4$ MIMO-CDMA system for PTBF, MES and GES, and much less 


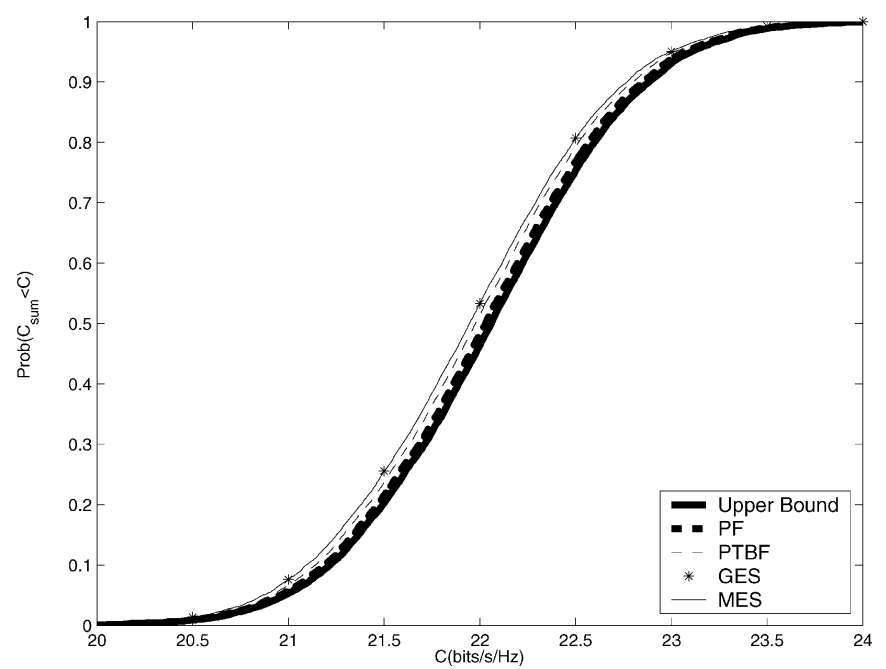

Fig. $8 . \quad K=12$ user MIMO-CDMA system with $4 \times 4$ MIMO model and $N=8$. Comparison of the sum capacity CDF curves of the upper bound and the MIMO-CDMA systems with different levels of feedback.

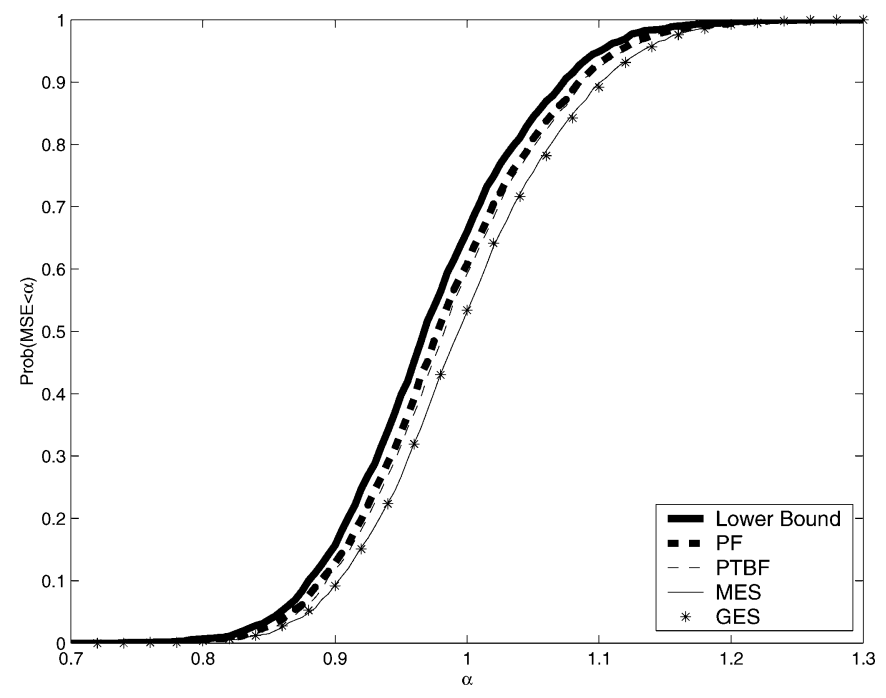

Fig. 9. $K=12$ user MIMO-CDMA system with $4 \times 4$ MIMO model and $N=8$. Comparison of the MSE CDF curves of the lower bound and the MIMO-CDMA systems with different levels of feedback.

for PF. Similarly, the difference between the achieved MSE and the lower bound is no more than 0.2 for $2 \times 2$ MIMO-CDMA system and 0.05 for $4 \times 4$ MIMO-CDMA system for PF, PTBF, MES and GES. As expected, the gap between the performance of proposed algorithms and the performance bounds decrease as the dimension of the MIMO system is increased. In Figs. 10 and 11, the performance of the algorithm for a multipath and asynchronous system where each user has 3 independent fading paths and a maximum delay of 2 chips is presented. We observed that the proposed algorithms perform close to the bounds for multipath asynchronous channels, as well.

\section{CONCLUDING REMARKS}

In this paper, we proposed signature and beamformer design algorithms for MIMO-CDMA systems with various levels of feedback. First, we investigated the problem assuming perfect feedback and derived iterative algorithms that enhance the

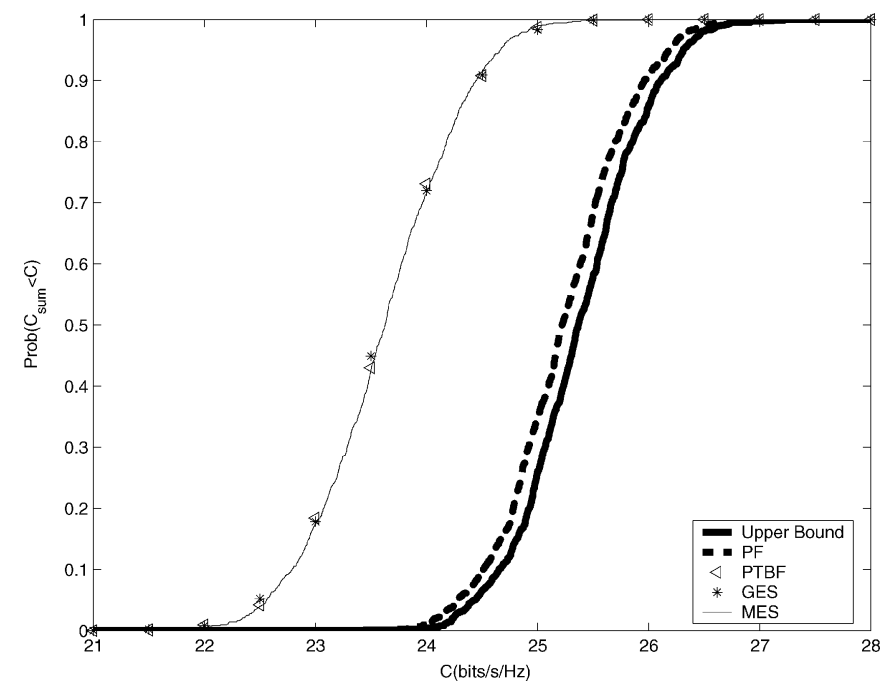

Fig. 10. $K=12$ user multipath and asynchronous MIMO-CDMA system with $4 \times 4$ MIMO model and $N=8$. Comparison of the CDF curves of the sum capacity upper bound and the achieved sum capacity with different levels of feedback.

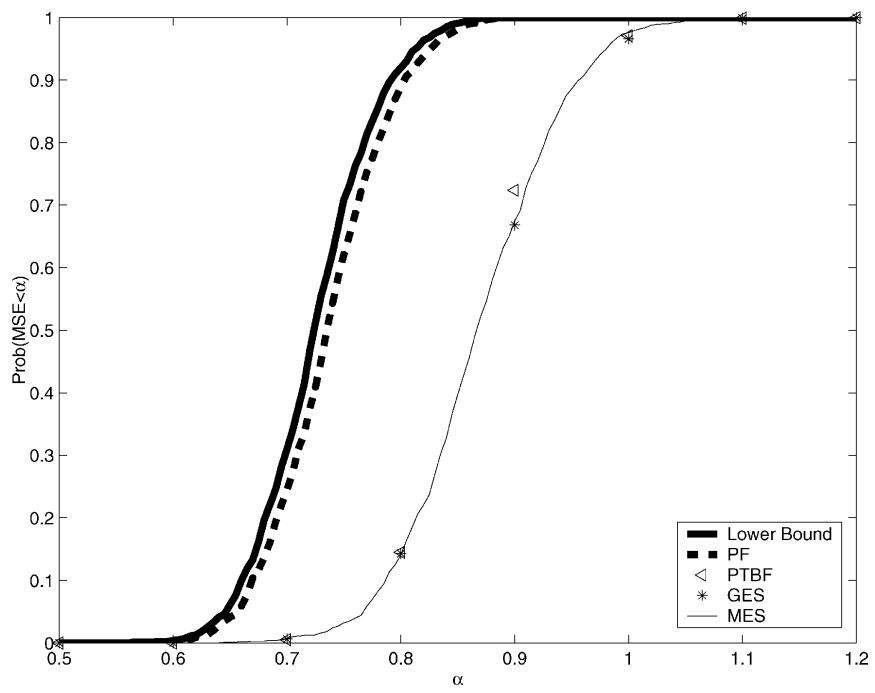

Fig. 11. $K=12$ user multipath and asynchronous MIMO-CDMA system with $4 \times 4$ MIMO model and $N=8$. Comparison of the CDF curves of the MSE lower bound and the achieved MSE with different levels of feedback.

system performance, i.e., the sum capacity, or the systemwide MSE. Next, motivated by limited feedback requirements, we investigated the orthogonal temporal signature assignment problem for given transmit beamformers and proposed a near-optimum signature assignment algorithm with low complexity. Since the performance of the system depends on the transmit beamformers, we have considered transmit beamformer selection next, and combined temporal signature assignment with several beamformers resulting from different levels of feedback at the transmitter side. We have observed that as the feedback level at the transmitter is increased, the performance of the proposed algorithms approaches to the performance bounds, and consequently to the performance of the jointly optimum signature and beamformer set. A complexity-performance trade-off is observed: more transmit side feedback prompts better performance at the expense of 
increased transmitter complexity. Notably, we observed that the individual CSI feedback facilitates a substantial gain with modest complexity requirements.

In this paper, we considered a system where the channels of the users are given, and the algorithms we propose rely on error-free, low-delay feedback channels. The effect of feedback accuracy remains to be investigated.

\section{REFERENCES}

[1] E. Telatar, "Capacity of multi-antenna Gaussian channels," Eur. Trans. Telecommun., vol. 10, no. 6, pp. 585-595, Nov./Dec. 1999.

[2] G. J. Foschini and M. J. Gans, "On limits of wireless communications in a fading environment when using multiple antennas," Wireless Personal Commun., vol. 6, no. 3, pp. 311-335, Mar. 1998.

[3] V. Tarokh, N. Seshadri, and R. Calderbank, "Space-time codes for high data rate wireless communication: Performance criterion and code construction," IEEE Trans. Inf. Theory, vol. 44, pp. 744-765, Mar. 1998.

[4] H. Sampath, P. Stoica, and A. Paulraj, "Generalized linear precoder and decoder design for MIMO channels using the weighted MMSE criterion," IEEE Trans. Commun., vol. 49, no. 12, pp. 2198-2206, Dec. 2001.

[5] S. Verdú, Multiuser Detection. Cambridge, U.K.: Cambridge Univ. Press, 1998.

[6] C. Rose, S. Ulukus, and R. D. Yates, "Wireless systems and interference avoidance," IEEE Trans. Wireless Commun., vol. 1, pp. 415-428, Jul. 2002.

[7] G. S. Rajappan and M. L. Honig, "Signature sequence adaptation for DS-CDMA with multipath," IEEE J. Sel. Areas Commun., vol. 20, pp. 384-395, Feb. 2002.

[8] A. F. Naguib, A. J. Paulraj, and T. Kailath, "Capacity improvement with base-station antenna arrays in cellular CDMA," IEEE Trans. Veh. Technol., vol. 43, pp. 691-698, Aug. 1994.

[9] A. Yener, R. D. Yates, and S. Ulukus, "Interference management for CDMA systems through power control, multiuser detection, and beamforming," IEEE Trans. Commun., vol. 49, no. 7, Jul. 2001.

[10] X. Wang and H. V. Poor, "Space-time multiuser detection in multipath CDMA channels," IEEE Trans. Signal Process., vol. 47, no. 9, pp. 2356-2374, Sep. 1999.

[11] S. Verdú, "Capacity region of Gaussian CDMA channels: The symbol-synchronous case," in Proc. 24th Annu. Allerton Conf. Communication, Control, Computing, Oct. 1986, pp. 1025-1034.

[12] M. Rupf and J. L. Massey, "Optimum sequence multisets for synchronous code-division multiple-access channels," IEEE Trans. Inf. Theory, vol. 40, pp. 1261-1266, Jul. 1994.

[13] S. Ulukus and R. D. Yates, "Iterative construction of optimum signature sequence sets in synchronous CDMA systems," IEEE Trans. Inf. Theory, vol. 47, pp. 1989-1998, Jul. 2001.

[14] S. Ulukus and A. Yener, "Iterative transmitter and receiver optimization for CDMA networks," IEEE Trans. Wireless Commun., vol. 3, pp. 1879-1884, Nov. 2004.

[15] W. Yu, W. Rhee, S. Boyd, and J. M. Cioffi, "Iterative water-filling for Gaussian vector multiple access channels," IEEE Trans. Inf. Theory, vol. 50, pp. 145-152, Jan. 2004.

[16] S. Serbetli and A. Yener, "Transceiver optimization for multiuser MIMO systems," IEEE Trans. Signal Process., vol. 52, no. 1, pp. 214-226, Jan. 2004.

[17] J. Rohani and L. Jalloul, "Orthogonal transmit diversity for direct-sequence spread CDMA," presented at the ETSI Special Mobile Group Plenary Meeting (SMG2), Stockholm, Sweden, Sep. 1997.

[18] B. Hochwald, T. L. Marzetta, and C. B. Papadias, "A transmitter diversity scheme for wideband CDMA systems based on space-time spreading," IEEE J. Sel. Areas Commun., vol. 19, pp. 48-60, Jan. 2001.

[19] P. Viswanath, V. Anantharam, and D. Tse, "Optimal sequences, power control and capacity of spread-spectrum systems with multiuser receivers," IEEE Trans. Inf. Theory, vol. 45, pp. 1968-1983, Sep. 1999.
[20] U. Madhow and M. L. Honig, "MMSE interference suppression for direct-sequence spread-spectrum CDMA," IEEE Trans. Commun., vol. 42, no. 12, pp. 3178-3188, Dec. 1994.

[21] B. Hassibi and B. M. Hochwald, "How much training is needed in multiple-antenna wireless links?," IEEE Trans. Inf. Theory, vol. 48, no. 4, pp. 951-963, Apr. 2003.

[22] S. Serbetli, S. Bethanabhotla, and A. Yener, "The effect of channel estimation on transceiver design for MIMO systems with QoS constraints," in Conf. Information Sciences Systems (CISS), Princeton, NJ, Mar. 2004.

[23] S. Serbetli and A. Yener, "Transmission strategies for correlated MIMO links with imperfect channel estimates," in IEEE Int. Conf. Communications (ICC), vol. 2, Seoul, Korea, May 2005, pp. 767-771.

[24] D. Bertsekas and J. Tsitsiklis, Parallel and Distributed Computation. Englewood Cliffs, NJ: Prentice-Hall, 1989.

[25] R. Zhang, "Scheduling for maximum capacity in S/TDMA systems," in Proc. IEEE Int. Conf. Acoustics, Speech, Signal Processing, 2002, pp. 2141-2144.

[26] G. Ausiello, P. Crescenzi, G. Gambosi, V. Kann, A. Marchetti-Spaccamela, and M. Protasi, Complexity and Approximation. New York: Springer-Verlag, 1999.

[27] D. S. Hochbaum, "Efficient bounds for the stable set, vertex cover and set packing problems," Discrete Appl. Math., vol. 6, pp. 243-254, 1983.

[28] M. M. Halldórsson, "Approximations of weighted independent set and hereditary subset problems," Proc. 5th Ann. Int. Conf. Computing Combinatorics, ser. Lecture Notes in Computer Science, pp. 261-270, 1999.

[29] R. S. Blum and J. H. Winters, "On optimum MIMO with antenna selection," IEEE Commun. Lett., vol. 6, pp. 322-324, Aug. 2002.

[30] A. F. Molisch, M. Z. Win, and J. H. Winters, "Capacity of MIMO systems with antenna selection," in Proc. Int. Conf. Communications, vol. 2, Helsinki, Finland, Jun. 2001, pp. 570-574.

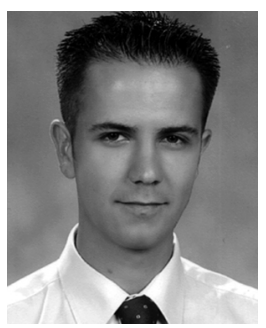

Semih Serbetli (S'02-M'05) received the B.S. degree in electrical and electronics engineering from Boğaziçi University, Istanbul, Turkey, in 2000, and the Ph.D. degree in electrical engineering from the Pennsylvania State University in 2005. His Ph.D. studies focused on transceiver design problems for multiuser multiple antenna systems.

He is currently with Philips Research Laboratories, Eindhoven, The Netherlands. His research interests include transceiver optimization for wireless communication systems with an emphasis on multiple-antenna (MIMO) systems, cooperative communications, and multicarrier communications systems.

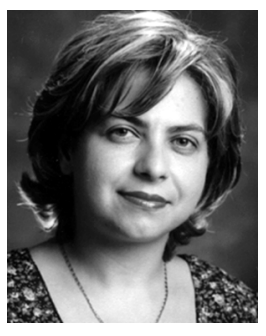

Aylin Yener (S'91-M'00) received the B.S. degrees in electrical and electronics engineering and in physics from Boğaziçi University, Istanbul, Turkey, in 1991 and the M.S. and Ph.D. degrees in electrical and computer engineering from Rutgers University, Piscataway, NJ, in 1994 and 2000, respectively.

During her Ph.D. studies, she was with Wireless Information Network Laboratory (WINLAB) in the Department of Electrical and Computer Engineering at Rutgers University. Between fall 2000 and fall 2001, she was with the Electrical Engineering and Computer Science Department at Lehigh University, PA, where she was a P.C. Rossin Assistant Professor. Currently, she is an Associate Professor with the Electrical Engineering Department at Pennsylvania State University, University Park. Her research interests include performance enhancement of multiuser systems, wireless communication theory, and wireless networking in general.

Dr. Yener is a recipient of the NSF CAREER award in 2003. She is an Associate Editor of the IEEE TRANSACTIONS ON WIRELESS COMMUNICATIONS. 